\title{
On the relation of the lunar recession and the length-of-the-day
}

\author{
Andre M. Maeder ${ }^{1} \cdot$ Vesselin G. Gueorguiev ${ }^{2,3}$
}

Received: 4 July 2021 / Accepted: 15 October 2021 / Published online: 29 October 2021

(c) The Author(s) 2021

\begin{abstract}
We review the problem of the consistency between the observed values of the lunar recession from $\mathrm{Lu}$ nar Laser Ranging (LLR) and of the increase of the lengthof-the-day (LOD). From observations of lunar occultations completed by recent IERS data, we derive a variation rate of the LOD equal to $1.09 \mathrm{~ms} / \mathrm{cy}$ from 1680 to 2020, which compares well with McCarthy and Babcock (Phys. Earth Planet. Inter. 44: 281, 1986) and Sidorenkov (Astron. Astrophys. Trans. 24: 425, 2005). This rate is lower than the mean rate of $1.78 \mathrm{~ms} / \mathrm{cy}$ derived by Stephenson et al. (Proc. R. Soc. A 472: 20160404, 2016) on the basis of eclipses in the Antiquity and Middle Age. The difference in the two observed rates starts at the epoch of a major change in the data accuracy with telescopic observations. The observed lunar recession appears too large when compared to the tidal slowing down of the Earth determined from eclipses in the Antiquity and Middle Age and even much more when determined from lunar occultations and IERS data from 1680 to 2020. With a proper account of the tidal effects and of the detailed studies on the atmospheric effects, the melting from icefields, the changes of the sea level, the glacial isostatic adjustment, and the core-mantle coupling, we conclude that the long-standing problem of the presence or absence of a local cosmological expansion is still an open question.
\end{abstract}

\footnotetext{
$\square$ A.M. Maeder

Andre.Maeder@UniGe.ch

V.G. Gueorguiev

Vesselin@MailAPS.org

1 Geneva Observatory, University of Geneva, chemin des Maillettes 51, 1290 Sauverny, Switzerland

2 Institute for Advanced Physical Studies, 21 Montevideo Street, Sofia 1618, Bulgaria

3 Ronin Institute for Independent Scholarship, 127 Haddon Pl., Montclair, NJ 07043, USA
}

Keywords Astrometry and celestial mechanics: eclipses-occultations - Planetary systems: Earth-Moon . Cosmology: Hubble-Lemaître expansion

\section{Introduction}

Since 1970, the Lunar Laser Ranging (LLR) provides direct measurements of the Earth-Moon distance with an initial accuracy of the order of few $\mathrm{dm}$ and gradually over 5 decades arrived at the present few-mm level. The transition to the order of $\mathrm{cm}$ levels was attained from the 80s to the early 90s, see for example (Williams et al. 2016). The results indicate the presence of lunar recession of $38.30 \pm 0.09 \mathrm{~mm} / \mathrm{yr}$. According to the formal definition from the International Earth Rotation and Reference Systems Service (IERS), the excess revolution time (with respect to a day $\Delta T$ of 86400 SI seconds) is called the length-of-the-day (LOD). We retain the standard notion to use LOD as the symbol for indicating the excess revolution time, while the change of the LOD is the derivative of $\Delta T$ with respect to time, it is usually determined in $\mathrm{ms} / \mathrm{cy}$. The relation between the lunar recession and the change of the LOD depends on a number of effects, astronomical, geophysical, climatic and atmospheric. Regarding the possibility of some additional contribution from cosmological expansion, Dumin (2016) points out that "It is quite surprising that many theorists believe that the possibility of local cosmological influences is strictly prohibited just by the available observational data, while a lot of observers believe that there are irrefutable theoretical proofs that Hubble-Lemaître expansion is absent at small scales." On both sides, the situation is still rich of uncertainties and this is what we try to study here.

The question whether astrophysical systems, such as the solar system and galaxies, follow the Hubble-Lemaître expansion has stimulated a vast literature since the pioneer 
work of McVittie $(1932,1933)$ and the Einstein-Straus theorem (Einstein and Straus 1945). The presence of an expansion at smaller scales has been considered as an open question by Bonnor (2000). The fact that the dark-energy dominates the matter-energy content of the Universe and that this energy appears as driving the acceleration of expansion is reviving the interest in the question: If dark energy is uniformly distributed in space would it not imply effects that may be present at small scales? The Earth-Moon system occupies a particular place in this context, since there are direct accurate measurements of the evolution of the distance in this two-body system.

In this respect, it is important to notice that the HubbleLemaitre expansion rate of the Universe, in century (cy) units is $H_{0} \approx 70(\mathrm{~km} / \mathrm{s}) / \mathrm{Mpc} \approx 0.7 \cdot 10^{-8} \mathrm{cy}^{-1}$. The recession rate estimated using the average Earth-Moon distance $d=384402 \mathrm{~km}$ that is increasing by $3.83 \mathrm{~cm} / \mathrm{yr}$ results in $\dot{d} / d=0.999 \cdot 10^{-8} \mathrm{cy}^{-1}$. While these two numbers may be regarded as a puzzling coincidence, the mystery plot thickens with two independent astrometric and radiometric Cassini data (Lainey et al. 2020) measurements of the mean recession speed of Titan from Saturn $v \approx 11.3 \mathrm{~cm} / \mathrm{yr}$, which when evaluated for the average Titan-Saturn distance $D=1221870 \mathrm{~km}$, results in $\dot{D} / D \approx 0.9 \cdot 10^{-8} \mathrm{cy}^{-1}$. The possibility of a scale-dependent Hubble constant has also been raised already by Krizek et al. (2015), Dumin (2018), Krizek et al. (2021). However, as it is well-known, the observed recession is currently thought to be only produced by the tidal interaction in the Earth-Moon system, which slows down the Earth rotation and thus produces an increase of the length-of-the-day. As this is the unique case where we have direct high accuracy and continuous measurements over centuries in the solar system, it is worth to examine closely the present status of the problem in relation with the above cosmological question.

In Sect. 2, we recall the basic relation between the LOD and the lunar recession imposed by the conservation of the total angular momentum in the classical theory. Section 3 examines the various theoretical predictions in the cosmological context. In Sect. 4, the different atmospheric and geophysical effects affecting the LOD are critically examined. In Sect. 5, the data sources on the LOD are collected and discussed, these are mainly the lunar and solar eclipses from the Antiquity and Middle Age, the lunar occultations of the past centuries and the data from the IERS. The analysis of the results is made in Sect. 6. Section 7 gives the conclusions.

\section{The LLR and its tidal relation with the length-of-the-day (LOD)}

The Earth-Moon tidal coupling produces some lunar recession observable by the Lunar Laser Ranging (LLR) exper- iment. The tidally distorted Earth rotates on its axis faster than the Moon on its orbit. Thus, the terrestrial deformation applies an additional forward attraction to the Moon, the angular momentum of which progressively increases at the expense of that of the Earth. Thus, the lunar recession is closely coupled to the slowing down of the Earth, i.e. the increase of the LOD. Many other effects also influence the LOD. For example, the Earth's moment of inertia changes due to the melting of polar icefields, the variations of the sea level and the isostatic rebound following deglaciation. There are exchanges of angular momentum between the solid Earth and atmosphere, the core and the mantle.

\subsection{The Lunar Laser Ranging (LLR)}

The first retroreflector on the Moon was placed in 1969 by Apollo 11, with 100 single reflectors with a diameter of $3.8 \mathrm{~cm}$. Successive improved reflectors were placed by the Apollo 14 and 15 flights (Dickey et al. 1994). The Apollo 15 retroreflector consists of 300 cube corners. With the reflector from the Lunokhod 2 rover (1973), a French array of 14 corner cubes of $11 \mathrm{~cm}$ on a Soviet rover, along with Lunokhod 1 which was lost but found in 2010 (Murphy et al. 2011), this makes a total of 5 reflectors. Short pulses of light are transmitted to the Moon and reflected back, the travel time being measured. The narrow initial beam is spread over an area of about $7 \mathrm{~km}$ diameter on the Moon, while the spot back on the Earth has a diameter of $20 \mathrm{~km}$. Thus, only a very small fraction of the photons (about $10^{-21}$ ) of the initial beam finally reaches the telescope back on the Earth. The first 15 years of the USA observations were conducted at McDonald Observatory, Texas. Observations were also performed in the Crimean Astrophysical Observatory with the retroreflectors installed on the Soviet rowers Lunokhod 1 and 2 launched in the early 70's, see Shiga (2010). The initial accuracy of distance measurements was $25 \mathrm{~cm}$, due to improvements from 1980 it went down to $2-3 \mathrm{~cm}$. In the 80's, new observing stations opened in Observatoire de la Côte d'Azur, France, and in Haleakala Observatory, Hawaii. The observations we mention below come from these observatories, as well as from Apache Point Observatory, New Mexico, and Matera, Italy. More recently new observing stations were developed in Hartebeesthoek-South Africa, Yunnan-China (meter-level accuracy), and Wettzell-Germany (mm-level accuracy), which considerably improve the accuracy. Between March 1970 and September 2015, there were 20138 ranges which led to an estimate of the lunar recession of $38.30 \pm 0.09 \mathrm{~mm} / \mathrm{yr}$ (Williams et al. 2016; Williams and Boggs 2016), a result that has not much changed since about three decades, since first determination by Christodoulidis et al. (1988). The geophysical model by Williams et al. associated to the determination of lunar orbital parameters predicts a corresponding increase of the LOD of $2.395 \mathrm{~ms} / \mathrm{cy}$ (millisecond per century). 


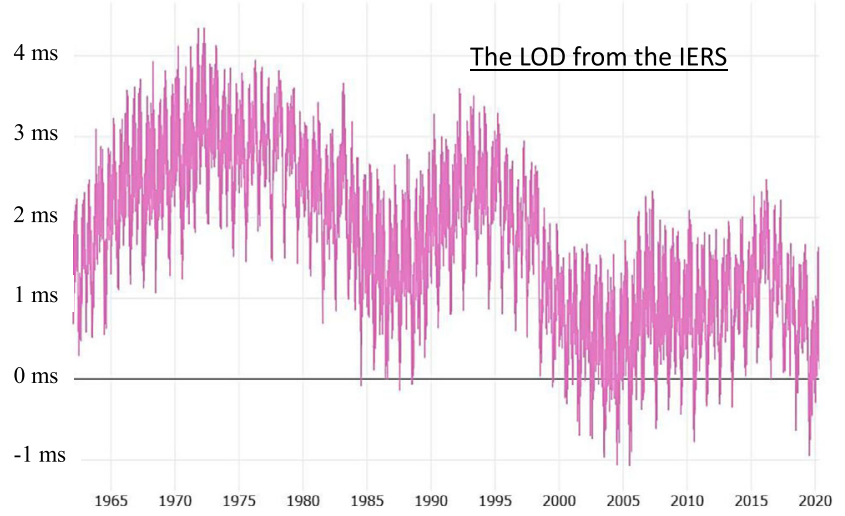

Fig. 1 The variations of the length of the day (on the left axis) in s as a function of time given in years from the International Earth Rotation Service (IERS 2020). The data cover the period of 1962 (with time obtained from atomic clocks) to the year 2020. The IERS data shown above exhibits a negative slope of $-0.0001 \pm 8.29 \times 10^{-7} \mathrm{~ms} / \mathrm{yr}$. We see the large seasonal variations and those over decades

\subsection{The LOD}

Since 1962 there are considerable improvements in the determination of the LOD and our geodetic knowledge. This is particularly due to the development of new techniques, in particular the space geodesy, SLR (Satellite Laser Ranging), GNSS (Global Navigation Satellite System), DORIS (Doppler Orbitography and Radio positioning Integrated by Satellite) and VLBI (Very Long Base Interferometry). The International Earth Rotation and Reference Frame System (IERS) (see the IERS URL to the Science/EarthRotation) is the recognized world basis for the study of the fundamental parameters of the Earth motion and orientation and the references frames.

Let us recall some key definitions. The time UT (Universal time) is defined by two consecutive crossings of the meridian by the Sun, this timescale depends on the Earth's rotation. It is different from the time TT (Terrestrial time) based on the SI second (International System) defined by the oscillations of the cesium atom in an atomic clock. A day in TT scale is defined by 86400 SI seconds. The difference $\Delta T=T T-U T$ gives the accumulated difference over a given period of time. The length-of-the-day (LOD), as already mentioned, is the the excess revolution time with respect to a day of 86400 SI seconds. The change of the LOD is the derivative of $\Delta T$ with respect to time, it is usually determined in ms/cy. Figure 1 above illustrates the LOD from 1962 to 2020 as given by the IERS. Given the already mentioned natural confusion due to the literal interpretation of the abbreviation LOD as Length of the Day, it should be stressed that it is a standard notion to use LOD as the symbol for indicating the excess revolution time, and as such, it is not anywhere close to 86400 SI seconds but more into ms range.

\subsection{Tidal effects}

Tides are due to the differential effects of gravitation on the various parts of a body. They depend on $R^{-3}$, where $R$ is the distance to the external source of gravitation generating the tide, see Tokieda (2013) for basics on tidal effects.

The tides produced by the Moon and the Sun are in an intensity ratio typically of 2.4 to 1 . There are dozens of tidal waves of different periods $P$ excited by the Moon and the Sun on the oceans, the Earth's crust and atmosphere (Simon et al. 2013). The designation of the tidal waves was defined by Georges Darwin in 1883 and is still in use. The main ones are the semi-diurnal tides which have the largest intensity coefficients (Lunar mean M2 P $=12.42 \mathrm{~h}$, solar mean S2 $\mathrm{P}=12.00 \mathrm{~h}$, lunar main elliptic $\mathrm{N} 2 \mathrm{P}=12.66 \mathrm{~h}$ ). The diurnal tides (Lunar principal $\mathrm{O} 1 \mathrm{P}=25.82 \mathrm{~h}$, lunar declination $\mathrm{K} 1$ $\mathrm{P}=23.93 \mathrm{~h}$, solar principal $\mathrm{P} 1 \mathrm{P}=24.07 \mathrm{~h}$ ) have a lower intensity, for example the ratio of $\mathrm{O} 1$ to $\mathrm{M} 2$ is $42 \%$. There are also the bimonthly (or fortnightly) tides excited by the Moon (Mf $\mathrm{P}=13.66$ days) with an intensity of $17 \%$ of $\mathrm{M} 2$. The lunar monthly tides ( $\mathrm{Mm} \mathrm{P}=27.55$ days) have an intensity of $9 \%$ of $\mathrm{M} 2$, the solar semi-annual ( $\mathrm{Ssa} \mathrm{P}=182.62$ days) an intensity of $8 \%$ of $\mathrm{M} 2$, the solar annual ( $\mathrm{Sa} \mathrm{P}=365.24$ days) an intensity of $1.3 \%$ of M2. Moreover, it is to be noted that a tidal wave, as for example M2, can be resolved in a spectrum of multiple nearby frequencies showing about a Gaussian distribution of intensities around the main component. Concerning the effects on the Earth-Moon distance, the main active tidal components are $\mathrm{M} 2, \mathrm{O} 1$ and $\mathrm{N} 2$, the semi-diurnal tides contribute to $88 \%$ of the effect, the diurnal ones to $13 \%$, while the Moon internal tides contributes to $1 \%$ with the opposite sign (Williams et al. 2016).

The tidal waves have complex figures on the sphere: zonal, sectorial, and tesseral (Simon et al. 2013). The oceanic and bodily tides are the most effective and also the main driving of the secular increase of the LOD and lunar recession. The amplitudes of the LOD variations, due to the M2 semi-diurnal oceanic tides are of the order of $\pm 1 \mathrm{~ms}$, as visible from Fig. 1 from the IERS. Following the semidiurnal tides, the fortnightly oceanic and crustal Mf tides which are zonal and symmetric with a period 13.66 day are also quite effective. A theoretical model, which accounts for 14 years of altimeter data from satellites (Ray and Egbert 2012), estimates that the amplitude of their effects on the LOD is about $0.36 \mathrm{~ms}$, a significant source of noise in the variations of the LOD around the mean in the IERS data. The oceanic and bodily tides produce monthly Mm variations of the LOD estimated to be about $\pm 0.30 \mathrm{~ms}$ (Sung-Ho et al. 2016). Let us mention that the crustal tides may reach up to half a meter at the equator, their effects on the LOD have been estimated to reach $0.1 \mathrm{~ms}$ by Defraigne and Smits (1999). 


\subsection{Calculation of the Earth-Moon tidal coupling}

The full system of equations describing the changes in the rotation of an elastic axisymmetric body having a fluid core and equilibrium oceans that is subject to small perturbing excitation has been rediscussed by Gross (2009). This system permits to study the changes of the Earth rotation produced by small changes in relative angular momentum or in the Earth's inertia tensor. Here, our aim is to connect quantitatively in a simple approach the lunar recession and the tidal braking of the Earth rotation. The main effect in the Earth (E)-Moon (M) system can be estimated by the angular momentum conservation of the whole system (Dumin 2003). This implies,

$$
\begin{aligned}
\cos \varphi I_{\mathrm{E}} \Omega_{\mathrm{E}}+M_{\mathrm{M}} R^{2} \Omega_{\mathrm{M}} & =\cos \varphi \sum_{i} I_{i} \Omega_{i}+M_{\mathrm{M}} R^{2} \Omega_{\mathrm{M}} \\
& =\text { const } .
\end{aligned}
$$

The index "E" refers to the global Earth, while the summation is made on the various parts $i$ of the rotating Earth: mantle, atmosphere and core. The angle $\varphi$, considered the same for the various rotating components, is the variable angle between the lunar orbital plane and the Earth equator. $M_{\mathrm{M}}$ is the mass of the Moon and $\Omega_{\mathrm{M}}$ its orbital angular velocity. $R$ is the mean distance between the Earth and Moon. We neglect the axial angular momentum of the Moon, since its mass $1.2 \%$ of that of the Earth and its axial rotation period (equal to its orbital period) is 27.3 days. Thus, the lunar axial angular momentum is a fraction of about $4 \cdot 10^{-4}$ of that of the Earth. As the angle $\varphi$ varies over the relatively short period of the lunar nodal precession (18.60 yr), we consider below a mean value of this angle.

$$
\begin{aligned}
& \cos \varphi\left[\sum_{i} I_{i}\left(\frac{\partial}{\partial t} \Omega_{i}\right)_{\mathrm{I}_{\mathrm{i}}}+\sum_{i} \Omega_{i}\left(\frac{\partial I_{i}}{\partial t}\right)_{\Omega_{i}}\right] \\
& +M_{\mathrm{M}} \frac{d}{d t}\left(R^{2} \Omega_{\mathrm{M}}\right)=0 .
\end{aligned}
$$

Account is given to the possible changes of the moment of inertia. The mean orbital angular velocity of the Moon is $\Omega_{\mathrm{M}}=G^{1 / 2} M_{\mathrm{E}}^{1 / 2} R^{-3 / 2}$, where $M_{\mathrm{E}}$ is the Earth's mass. The previous equation becomes, if $T_{\mathrm{i}}=2 \pi / \Omega_{\mathrm{i}}$ is the period of the axial rotation of the i-component (a day for the mantle),

$$
\begin{aligned}
& 2 \pi \cos \varphi\left[-\sum_{i} I_{i} \frac{1}{T_{i}^{2}}\left(\frac{\partial T_{i}}{\partial t}\right)_{\mathrm{I}_{\mathrm{i}}}+\sum_{i} \frac{1}{T_{i}}\left(\frac{\partial I_{i}}{\partial t}\right)_{\mathrm{T}_{\mathrm{i}}}\right] \\
& +G^{1 / 2} M_{\mathrm{M}} \frac{M_{\mathrm{E}}^{1 / 2}}{2 R^{1 / 2}} \frac{d R}{d t}=0 .
\end{aligned}
$$

This equation can be written as,

$$
\frac{d R}{d t}=\sum_{i}\left[k_{i}\left(\frac{\partial T_{i}}{\partial t}\right)_{\mathrm{I}_{\mathrm{i}}}-k_{i}^{\prime}\left(\frac{\partial I_{\mathrm{i}}}{\partial t}\right)_{\mathrm{T}_{\mathrm{i}}}\right] \text {, }
$$

with $k_{i}$ and $k^{\prime}$ defined by

$k_{i}=\frac{I_{i}}{T_{i}} k_{i}^{\prime} \quad$ and $\quad k_{i}^{\prime}=4 \pi \cos \varphi \sqrt{\frac{R}{T_{\mathrm{i}}^{2} G M_{\mathrm{M}}^{2} M_{\mathrm{E}}}}$.

Equation (4) expresses the lunar recession as a function of the change of rotation period of the Earth's components and the change of the moments of inertia. If we consider only the tidal interaction between the Earth globally and the Moon, we get similarly for a constant global moment of inertia $I_{\mathrm{E}}$,

$\frac{d R}{d t}=k_{\mathrm{E}} \frac{d T_{\mathrm{E}}}{d t}, \quad$ with $k_{\mathrm{E}}=4 \pi \cos \varphi \frac{R^{1 / 2} I_{\mathrm{E}}}{T_{\mathrm{E}}^{2} G^{1 / 2} M_{\mathrm{M}} M_{\mathrm{E}}^{1 / 2}}$.

Thus, $\frac{d T_{\mathrm{E}}}{d t}$ is the Earth's slowing down due to the tidal interaction. We mentioned above that a complex model of the tidal interaction leads to an estimate of the increase of the LOD of $\frac{d T_{\mathrm{E}}}{d t}=2.395 \mathrm{~ms} / \mathrm{cy}$, corresponding to the observed lunar recession of $\frac{d R}{d t}=3.83 \mathrm{~cm} / \mathrm{yr}$ (Williams et al. 2016; Williams and Boggs 2016). We adopt the following numerical values:

$$
\begin{aligned}
& M_{\mathrm{E}}=5.973 \cdot 10^{27} \mathrm{~g}, \quad R_{\mathrm{E}}=6.371 \cdot 10^{8} \mathrm{~cm}, \\
& M_{\mathrm{M}}=7.342 \cdot 10^{25} \mathrm{~g}, \quad R=3.844 \cdot 10^{10} \mathrm{~cm}, \\
& I_{\mathrm{E}}=0.331 \cdot M_{\mathrm{E}} R_{\mathrm{E}}^{2}=8.0184 \cdot 10^{44} \mathrm{~g} \mathrm{~cm}^{2} .
\end{aligned}
$$

The numerical coefficient 0.331 is obtained from precession data by Williams (1994). The angle $\varphi$ varies between 18.16 and 28.72 degrees, thus we adopt a mean value of $\cos \varphi=0.914$. The coefficient $k_{\mathrm{E}}=1.650 \cdot 10^{5} \mathrm{~cm} \mathrm{~s}^{-1}$. For $k_{i}^{\prime}$, taking $T_{i} \equiv T_{\mathrm{E}}$, we get $k_{i}^{\prime}=1.777 \cdot 10^{-35} \mathrm{~g}^{-1} \mathrm{~cm}^{-1}$. With the appropriate units used in the study of the LLR, we write Eq. (6),

$\frac{d R}{d t}\left[\frac{\mathrm{cm}}{\mathrm{yr}}\right]=1.650 \cdot 10^{5}\left[\frac{\mathrm{cm}}{\mathrm{s}}\right] \frac{d T_{\mathrm{E}}}{d t}\left[\frac{\mathrm{s}}{\mathrm{yr}}\right]$.

This expresses the tidal relation between the lunar recession and the increase of the axial rotation period $T_{\mathrm{E}}$ of the Earth, i.e. the LOD, in absence of other effects. An estimate based on the above expression (8), applied to the observed Moon recession, gives an increase of the LOD of $2.32 \cdot 10^{-5}$ $\mathrm{s} \mathrm{yr}^{-1}$, a value remarkably close (at $3.1 \%$ ) to that derived from a more detailed model. This means that the angular momentum conservation effectively contains the resulting pull of the numerous tidal waves and that the approximations made have a limited incidence. Mechanical effects in the Earth-Moon system have an "instantaneous" action. Let us consider the Earth-Moon system as a two-body isolated system.

The best way to assess the possible effect of the Hubble expansion (cf. Sect. 3) is via the comparison of the observed 
lunar recession to the anticipated tidal effects. If there is an additional cosmological contribution to the lunar recession, the total observed recession $\left(\frac{d R}{d t}\right)_{\mathrm{obs}}$ is the sum of two contributions, the effectively tidal one $\left(\frac{d R}{d t}\right)_{\text {tdeff }}$ and the cosmological one $\left(\frac{d R}{d t}\right)_{\text {cosm }}$. Thus, one has

$$
\left(\frac{d R}{d t}\right)_{\mathrm{tdeff}}=\left(\frac{d R}{d t}\right)_{\mathrm{obs}}-\left(\frac{d R}{d t}\right)_{\operatorname{cosm}} .
$$

This expression will be used in establishing Table 1 below.

Equation (6) which expresses the global tidal coupling, can be written as $\left(\frac{d T_{\mathrm{E}}}{d t}\right)_{\text {tdeff }}=\frac{1}{k_{\mathrm{E}}}\left(\frac{d R}{d t}\right)_{\text {tdeff }}$ that is corresponding to the effective tidal change of the Earth's rotation period and thus is given by Eq. (4) and leads to:

$k_{\mathrm{E}}\left(\frac{d T_{\mathrm{E}}}{d t}\right)_{\text {tdeff }}=\sum_{i}\left[k_{i}\left(\frac{\partial T_{i}}{\partial t}\right)_{\mathrm{I}_{\mathrm{i}}}-k_{i}^{\prime}\left(\frac{\partial I_{\mathrm{i}}}{\partial t}\right)_{\mathrm{T}_{\mathrm{i}}}\right]$.

The above equation allows us to distinguish between the effects producing exchanges of angular momentum from those which modify the Earth's moment of inertia. The atmospheric motions (circulation currents, zonal winds, etc.) produce exchanges of angular momentum between the atmosphere and the mantle, other exchanges also occur between the mantle and the core. Thus, we consider below in a simplified way three " $i$ " components of the Earth: the atmosphere (atm), the mantle (mtl) and the core (cr), which may all experience changes of rotation periods. Thus, the above expression can be rewritten

$$
\begin{aligned}
\frac{d T_{\mathrm{mtl}}}{d t}= & \frac{k_{\mathrm{E}}}{k_{\mathrm{mtl}}}\left(\frac{d T_{\mathrm{E}}}{d t}\right)_{\mathrm{tdeff}}-\frac{k_{\mathrm{atm}}}{k_{\mathrm{mtl}}} \frac{d T_{\mathrm{atm}}}{d t}-\frac{k_{\mathrm{cr}}}{k_{\mathrm{mtl}}} \frac{d T_{\mathrm{cr}}}{d t} \\
& +\sum_{i} \frac{k_{i}^{\prime}}{k_{\mathrm{mtl}}} \frac{d I_{\mathrm{i}}}{d t},
\end{aligned}
$$

with the condition that for each derivative the other factors are considered as constant. The ratios of the k-terms express the weighting according to the moments of inertia, and assuming an equality of the periods in the various k-factors, one has:

$$
\begin{aligned}
\frac{k_{\mathrm{E}}}{k_{\mathrm{mtl}}} & \approx \frac{I_{\mathrm{E}}}{I_{\mathrm{mtl}}} \approx 1.013, \quad \frac{k_{\mathrm{atm}}}{k_{\mathrm{mtl}}} \approx \frac{I_{\mathrm{atm}}}{I_{\mathrm{mtl}}} \approx 1.7 \cdot 10^{-6}, \\
\frac{k_{\mathrm{cr}}}{k_{\mathrm{mtl}}} & \approx \frac{I_{\mathrm{cr}}}{I_{\mathrm{mtl}}} \approx 0.013 .
\end{aligned}
$$

For estimates, we use a core mass equal to $32.5 \%$ of that of the Earth with a radius of $1217.5 \mathrm{~km}$ (Kennett et al. 1995), with a numerical coefficient of 0.36 to obtain $I_{\mathrm{cr}}$. We have $I_{\mathrm{mtl}}=I_{\mathrm{E}}-I_{\mathrm{cr}}$ and a mass of the atmosphere of $5.15 \cdot 10^{21} \mathrm{~g}$. The term on the left in Eq. (11), giving the change of the rotation period of the mantle, corresponds to the observed change $\dot{\tau}_{\text {obs }}$ of the LOD, which is expressed in ms/cy. The first term on the right is the change $\dot{\tau}_{\text {tdeff }}$ of the LOD corresponding to the effective lunar recession, the cosmological effect being subtracted, if anyone. The correspondence is based on the fact that a lunar recession of $3.83 \mathrm{~cm} / \mathrm{yr}$ is related to a change of the LOD of $2.395 \mathrm{~ms} / \mathrm{cy}$. The second and third term on the right are respectively the atmospheric $\dot{\tau}_{\text {atm }}$ and core $\dot{\tau}_{\text {cr }}$ contributions. They allow us to express the effects of the exchange between the solid Earth with the atmosphere and the core. Thus, if $T_{\text {atm }}$ or $T_{\mathrm{cr}}$ increases, the angular momentum conservation implies that the observed change of the LOD should be reduced. Any increase of the moment of inertia of the Earth would increase the LOD. Changes occur in particular by melting of high latitude icefields and by the resulting changes of the sea level, as well by the isostatic rebound also called Global Isostatic Adjustment (GIA). Finally, we may write

$\dot{\tau}_{\mathrm{obs}}=\dot{\tau}_{\mathrm{tdeff}}+\dot{\tau}_{\mathrm{atm}}+\dot{\tau}_{\mathrm{cr}}+\dot{\tau}_{\mathrm{ice}}+\dot{\tau}_{\mathrm{sea}}+\dot{\tau}_{\mathrm{GIA}}$,

where each term represents a specific contribution to the time variation of the LOD expressed in ms/cy: terms $\dot{\tau}_{\text {atm }}$ and $\dot{\tau}_{\text {cr }}$ for the exchanges of the mantle with atmosphere and core, the next ones for the change of the moment of inertia due to ice melting, change of the sea level and glacial isostatic adjustment respectively. Thus, we verify that the angular momentum conservation allows the addition, properly weighted by the moment of inertia, of the various characteristic times. An expression of that sort has been written by Munk (2002). Now, the great challenge is to get the numerical values of the terms contributing to this equation.

The tidal effects can also be theoretically calculated from the predicted tidal distortions of the Earth's atmosphere, oceans, and mantle. There appear some problem here. Globally, these distortions would lead to a much smaller tidal effects than the one anticipated from the lunar recession. They would only account for about $55 \%$ of the observed lunar recession of $3.83 \mathrm{~cm} / \mathrm{yr}$, as emphasized by Krizek et al. (2015) on the basis of the several studies. This was first mentioned by van Flandern (1975). Also, the disagreement between the rate of lunar recession and the LOD variation was attributed to a possible cosmological expansion by Dumin (2001). The tidally deformed figure of the Earth has also been discussed by Novotny (1998) and further analysed in the geophysical studies of the history of the Earth's rotation by Peltier (2007).

\subsection{The Moon's orbital motion}

The assumption of a circular orbital motion of the Moon is a crude assumption. The orbit is an ellipse with eccentricity of 0.055 , so that the distance between the perigee and apogee varies by about $43000 \mathrm{~km}$ with a synodic period of 


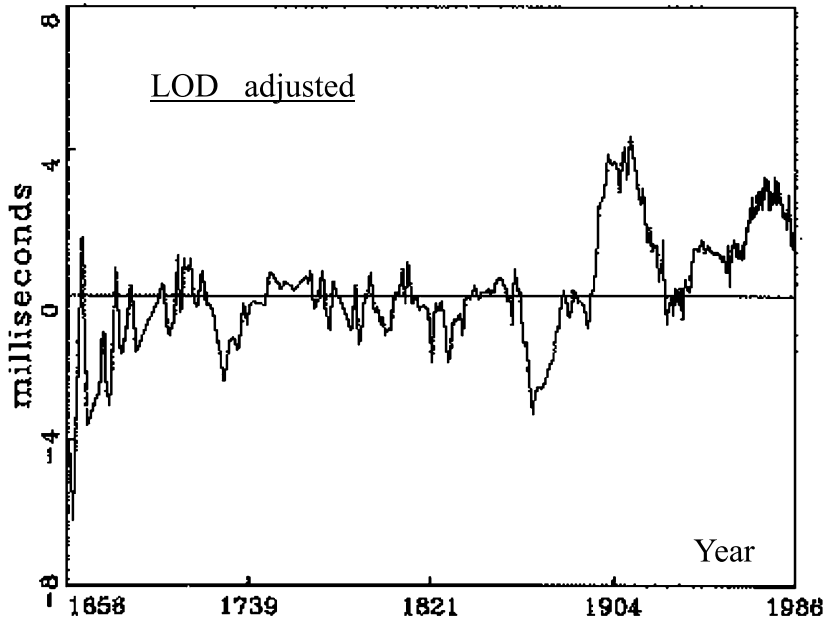

Fig. 2 Graphic representation of the data by McCarthy and Babcock (1986) on the variations of the LOD since 1656. The values have been adjusted by these authors for the mean decrease of the angular velocity of the Earth given in Eq. (19). This way the mean trend of $+0.73 \mathrm{~ms} / \mathrm{cy}$ appears to be at zero - the horizontal line of the Figure from McCarthy and Babcock (1986)

29.53 days. A number of other effects in the lunar motion intervene: the apsidal precession (change of the orientation of the semi-major axis of the elliptic lunar orbit with a period of $8.85 \mathrm{yr}$ ); the nodal precession (related to the precession of the orbital plane of the Moon), as a consequence the angle $\varphi$ between the lunar orbital plane and the Earth equator varies with a period $18.60 \mathrm{yr}$, as seen above. Some of the secondary oscillations of the LOD in Figs. 1, 2, and 5 may be due to the apsidal and nodal precession. There are also the axial precession of the Moon, its physical libration and nutation which have more limited effects (Dehant and Mathews 2009). The precession of the Earth is acting on a much longer timescale of $26000 \mathrm{yr}$.

\section{Cosmology and expansion at small scales}

The question arises whether there are also some small additional effects of the global cosmological expansion locally at small scales. Following McVittie (1933), most authors were studying this problem with a metric combining an exterior Schwarzschild solution of a mass concentration, and a FLRW metric representing the expanding Universe. For a review on the attempts to estimate the influence of the cosmological expansion on local systems, see Carrera and Giulini (2010). Most authors generally concluded to no effect like Williams et al. (2004), or a negligible effect, see Cooperstock et al. (1998) and Sereno and Jetzer (2007). Anderson (1995) found some expansion of small systems, but at a negligible rate. Price and Romano (2005) proposed "the all or nothing" expansion, a work discussed by Faraoni and Jacques (2007), who pointed out that it applies to a de Sit- ter background, towards which the Universe is tending. The absence of an effect is also more a postulate, not to say a dogma, in some major references, see Misner et al. (1973). These authors also postulated that Hubble expansion must be truncated at some sufficiently small scale, because otherwise a unit of length could not be defined by which the expansion can be measured. However, there are a few different voices. Bonnor (2000) considered that: "The scale at which the cosmic expansion begins to affect objects seems to be an open question. Intuitively it is hard to think of objects held together by strong forces, such as those of the electric field, as taking part in the Hubble expansion. However, cosmologists often say the expansion is that of space itself. From this point of view it would not be so counter intuitive if ordinary bodies expanded with the universe." Ordinary bodies, like crystals, atoms, and nuclei do not expand due to the predominance of non-gravitational forces like electromagnetic and strong force. We should deal only with free bodies that interact gravitationally.

In the theorem by Einstein and Straus (1945), the authors consider a central mass $M=\frac{4 \pi \varrho R^{3}}{3}$ made by the concentration of matter with a mean density $\varrho$ from a spherical cavity of radius $R$, embedded in an expanding Universe. The theorem states that there is no expansion in the empty cavity, while beyond it, including the limit $R$, the expansion is present, operating according to the Hubble law. In a simplified way, the argument means that the limit between expansion and no expansion occurs when the kinetic energy $(1 / 2) v^{2}=(1 / 2)(H R)^{2}$ of the expansion is equal to the local potential energy $G M / r$ for a spherical geometry (taking the Virial expression or not makes no big difference). This corresponds to $H=\sqrt{\frac{8 \pi}{3} G \varrho}$, the standard expression of the Hubble rate in Friedman cosmology for a flat Universe. Thus, inside the cavity, no expansion is predicted. This is the global sense of the Einstein-Straus theorem and of various approaches. The fact that there is an abrupt change from no expansion to full expansion when crossing radius $R$, while gravity is a continuously varying function, is very disconcerting, at least for the present authors.

Nowadays the case of the Einstein-Straus theorem is even more problematic, since the Universe is dominated by dark energy, which represents about $70 \%$ of the matter-energy and is the driving force of the accelerated expansion. As dark energy is the same everywhere, the conditions of the Einstein-Straus theorem are never met: there should be no empty cavity free from mass-energy, and thus without expansion in the $\Lambda \mathrm{CDM}$ model, for more in depth discussion and critics of no-expansion claims see the original work by Dumin (2005).

The effects of the cosmological constant $\Lambda$, which expresses the energy-density of the vacuum, have been considered by Balbinot et al. (1988), Klioner and Soffel (2005) and Sereno and Jetzer (2007). The conclusions were that there 
is some expansion, but the effects were found insignificant, for example Sereno and Jetzer (2007) estimate an expansion of the Earth-Sun distance with a rate of $10^{-21} \mathrm{~m} / \mathrm{yr}$. There is a noticeable exception with Dumin (2016, 2020), who applies a new metric inspired from the original Kottler metric (Kottler 1918), which accounts for a central mass (cf. Schwarzschild metric) and the $\Lambda$-term (cf. de Sitter metric). Dumin makes some further transformation of the metric to account for expansion. He obtains outwards spiraling orbits and conclude to the existence of a slightly smaller local Hubble expansion than the global one. Thus, on the whole the conclusions of standard models are generally in favor of a negligible effect of the Hubble expansion in planetary systems. Nevertheless, important divergent claims are also expressed (Bonnor 2000; Dumin 2016).

The work of Klioner and Soffel (2005) concludes that cosmological effects for solar system bodies are negligible over the age of the Universe. However, their imbedding of the Barycentric Celestial Reference System into a cosmological background considers only a central perturbation potential. While such a perturbation due to a central potential could be used to probe dark matter effects in the solar system, it seems inadequate for probing a cosmic expansion effects that should not have a central point. The inadequacy of central potentials for understanding very low Newtonian gravity limits has been discussed previously by Maeder and Gueorguiev (2020a).

Several non-standard cosmological models are exploring new research lines, stimulated by the fact that about $95 \%$ of the mass-energy in the Universe lies in unknown dark components. In the framework of scale invariant models, a general scale invariant field equation has been obtained (Canuto et al. 1977) in the line of developments made by Dirac (1973). The scale invariant vacuum (SIV) theory rests on the specific hypothesis that the macroscopic empty space is invariant to a scale transformation $d s^{\prime}=\lambda(t) d s$, a property present in Maxwell's equation of electrodynamics, as well as in General Relativity in absence of a cosmological constant, see review by Maeder and Gueorguiev (2020b). Cosmological equations have been obtained leading to accelerated Universe models (Maeder 2017). The weak field approximation of the geodesic equation (Dirac 1973; Bouvier and Maeder 1978) allows the study of the two-body problem, which predicts that the semi-major axis $a$ increases like $\dot{a} / a=1 / t$, where $t$ is the time in the timescale of some cosmological models, thus the rate of expansion of a two-body system is not necessarily the observed Hubble expansion rate, although it is likely of the same order of magnitude. While the MOND theory (Milgrom 1983, 2019) considers a dilatation invariance with a constant scale factor, the SIV theory assumes a scale factor dependent on time. The original weakfield approximation in the SIV utilized the assumption of isotropic and homogeneous space for the derivation of the terms beyond the usual Newtonian equations (Dirac 1973; Bouvier and Maeder 1978). The result is a velocity dependent extra term $\kappa_{0} \vec{v}$ with $\kappa_{0}=1 / t$. Recently, similar term was derived as a result of un-proper time parametrization (Gueorguiev and Maeder 2021). Krizek et al. (2015) are emphasizing that there is no reason that the dark energy responsible for the accelerated expansion does not manifest itself in galaxies and in the Solar System as well. They examine a number of astrophysical problems, including the lunar recession, from which they suggest that even bound systems expand at a rate of the order of the Hubble expansion.

There are several other Extended Theories of Gravity (Capozziello and de Laurentis 2011), where some modifications of the dynamics are predicted. The so-called $f(R)$ theories consider a gravity Lagrangian, modified by including higher order terms depending on curvature $R$ (Capozziello et al. 2006; Capozziello and Stornaiolo 2008). The deformations act like a force that deviates the test particles. The geodesics and general field equations are consistent with those by Canuto et al. (1977). Sola et al. (2015) consider a dynamical vacuum, with $\Lambda(H)=C_{0}+C_{\mathrm{H}} H^{2}+C_{\dot{\mathrm{H}}} \dot{H}$, where the time dependence of $\Lambda$ improves a number of cosmological tests (Sola et al. 2015, 2017). Meierovich (2012, 2013) uses a longitudinal vector field to represent the effects of the dark components in the framework of GR. In this respect, there are some similarities with the SIV. The analysis below is independent of any cosmological models.

\section{Changes of the LOD due to various terrestrial effects}

The richness of the terrestrial effects influencing the LOD as they appear in Eq. (13) is impressive. Each one corresponds to a major current research field. Here we attempt to briefly report on the main effects, their timescales and amplitudes.

\subsection{Atmospheric effects on the Earth's rotation and the LOD}

The atmosphere has an influence on the Earth's spin rotation, by the exchanges of angular momentum between the solid Earth and the atmosphere. The oscillation amplitudes grow exponentially with altitude in the rarefied gas. The exchanges are generated by atmospheric tides and also by winds and global circulation currents in the atmosphere. The atmospheric effects on the LOD have a limited amplitude. Globally, the atmosphere super-rotates with an average velocity of $7 \mathrm{~m} / \mathrm{s}$ with respect to the solid Earth. If it would stop super-rotating and just co-rotate with the Earth, the LOD would change by $3 \mathrm{~ms}$ accounting for the respective moments of inertia (Gross 2009). This means that the large cyclic variations observed in the LOD (the so-called decadal 
and the 1500 yr-oscillations) cannot be due to exchanges between the Earth's mantle and atmosphere. The timescales for these exchanges are ranging from half a day to a few years, for a recent review see Sung-Ho et al. (2016).

Let us start by the short timescales. The intense semidiurnal atmospheric tide S2 (the major solar tidal component) has a peculiar effect. While the oceanic lunar and solar tides are excited by the gravitational differential pull, the semi-diurnal atmospheric tides are first excited by the Sun heating. The maximum atmospheric pressure occurs in general around 10 a.m. local time, i.e. before the Sun reaches the zenith, thus the solar attraction exerts an additional pull on the present excess of atmospheric matter. This means that the main component of the solar atmospheric tides has an effect opposed to that of the Moon by accelerating the Earth's spin rotation, an effect first found by Munk and MacDonald (1960). The energy exchange due to this effect has been re-estimated to be about $4.5 \%$ of that of the tidal energy dissipation of oceans and mantle (Sung-Ho et al. 2016). Since the energy of the rotation depends on the square of the pe$\operatorname{riod} T_{\mathrm{E}}$, the effect on the LOD is about $2.3 \%$ of that due to the total tidal energy dissipation. This means that the semidiurnal solar tide accelerates the Earth rotation by something like $0.05 \mathrm{~ms} / \mathrm{cy}$. This small effect is often not accounted for.

The atmospheric effects have been studied by a number of authors over the last decades, see for example Eubanks et al. (1985), Hay et al. (2016) and Sung-Ho et al. (2016). Let us follow these last authors, who express the equations for the effects of the pressure distribution on tides and make a quantitative analysis of the various terms. They have also used complete datasets of $6 \mathrm{hr}$ interval global atmospheric pressure and wind velocity since 1980 from the ECMWF (The European Centre for Medium-Range Weather Forecasts). On this basis, they have performed comparisons between the meteorological changes and the variations of the LOD, as obtained from the IERS website. There is a strong connection between the change of the atmospheric angular momentum and the LOD, which results from surface friction by the winds, particularly the zonal winds, with a characteristic timescale of about 7 days (Hay et al. 2016). Sung-Ho et al. (2016) confirm the seasonal variations of the LOD, which are due to changes in the global atmospheric circulation. These effects, accompanied by polar drifts, are relatively large, the LOD is shorter from late June to early September by $-0.65 \mathrm{~ms}$, and longer by $+0.65 \mathrm{~ms}$ from Winter to Spring time (Sung-Ho et al. 2016), see also Fig. 1.

The atmospheric interactions also have timescales of years, as in the case of El-Niño-Southern-Oscillation (ENSO). Gross (2016) and Schindelegger et al. (2017) estimate that about $30 \%$ of the time-averaged rotation rate of the atmospheric contributions may be linked in some way to ENSO. According to Sidorenkov (1997), most of the periods in the ENSO component spectrum $(6,3.6,(2.8), 2.4 \mathrm{yr})$, except for 2.8, are multiple of the Chandler period of 1.2 $\mathrm{yr}$, (the Chandler wobble is a small nutation of the Earth's rotation axis of $9 \mathrm{~m}$ amplitude). Most fluctuations of the LOD in range of 40 to 700 days are due to atmospheric interactions Eubanks et al. (1985). On the longer term, there is an equilibrium between the atmospheric pick-up of angular momentum at low and high latitudes and its transfer to the Earth by West winds at middle latitudes (Hay et al. 2016).

Summary on atmospheric effects on the LOD: On a timescale of more than three centuries, as we are considering in this study, the positive and negative atmospheric effects on the change of the LOD largely cancel each other on much shorter timescales. Thus, we must only account for the acceleration of the Earth rotation due to the semi-diurnal solar atmospheric tides S2, for which a contribution of $-0.05 \mathrm{~ms} / \mathrm{cy}$ to the LOD was estimated here.

\subsection{Effects of ice melting and change of the sea level}

The melting of glaciers and polar ice-fields and the resulting increase of the sea level have sizable consequences for the Earth's spin rotation rate, as well as for the polar wander of the rotation axis, see review by Mitrovica and Wahr (2011). The case of polar ice-fields is particularly critical at the present time, their strong melting since the year 1990 leads to an increase of the sea level over the world. Before 1990 , the increase of sea level is mainly due to the melting of glaciers outside from the polar caps. Melting implies a global motion of water away from the polar axis, producing a growth of the Earth's moment of inertia, and thus an increase of the LOD. In a pioneer work, Munk and MacDonald (1960) estimated the relation between the changes of polar ice caps, the sea level and the length-of-the-day: globally, a $1 \mathrm{~cm}$ eustatic rise (change of the global ocean mass) of the sea level is leading to a change of the LOD equal to $+0.1 \mathrm{~ms}$, see also Munk (2002)

In the context of global warming, an important melting produces a rapid increase of the sea level. This is true nowadays, but the reality for the 20th century is rather different. The global balance between the ice accumulation in polar regions and calving was about zero as suggested in many works by Radok et al. (1987), Trupin (1993), Johnston and Lambeck (1999), Munk (2002) and the report of the IPCC 2001. The polar studies by Radok et al. (1987) showed that the ice sheets of Antarctica and Greenland (the two major icefields) are in equilibrium between accumulation and discharge, so that the net change is about zero. Gridded estimates of ice accumulation on the Antarctic showed an accumulation of ice of $4.5 \mathrm{~cm} / \mathrm{yr}$ in the center of the continent to about $50 \mathrm{~cm} / \mathrm{yr}$ at the edge. On Greenland there was evidences of a thickening in the years 1950-1970. Thus, these two major polar icefields could even have contributed 
to a reduction of the Earth's moment of inertia in the 20th century (Trupin 1993). Johnston and Lambeck (1999), in a study on the change of the oblateness term in the expression of the Earth potential, also considered the ice accumulation and ablation from Antarctica and Greenland to be about equivalent within $\pm 10 \%$. This result for these two sites is also confirmed by the 2001 report of the IPCC, which gives a value of $-2 \mathrm{~cm} / \mathrm{cy}$ for water storage and an effect of $+3 \mathrm{~cm} / \mathrm{cy}$ for ice melting, in equivalent effect on the sea level (the estimates for Greenland are $0.5( \pm 0.5) \mathrm{mm} / \mathrm{yr}$ and for Antarctica $0.5( \pm 1.0) \mathrm{mm} / \mathrm{yr})$. Even more constraining, a rise of sea level of $0.7( \pm 0.1) \mathrm{mm} / \mathrm{yr}$, due melting in polar regions for the period 1900-1990, would lead to disagreement with Lageos I satellite determinations of the geopotential according to Mitrovica et al. (2015). The change of the sea level for the period 1900-1990 appears essentially not due to mass loss from polar icefields (up to a maximum of $0.2 \mathrm{~mm} / \mathrm{yr}$ ), but to the melting of other glaciers and water thermal dilatation (Mitrovica et al. 2015). Year 1990 marks the start of a high mass flux from polar regions, as well as an increase of the fast melting of mountain glaciers.

The eustatic contribution was estimated in 2001 by the Intergovernmental Panel on Climate Changes (IPCC) to correspond to $6 \mathrm{~cm} / \mathrm{cy}$, to which a thermal dilatation effect of $3 \mathrm{~cm} / \mathrm{cy}$ must be added (Munk 2002). Often previous estimates suggested a rise of the sea level of 1.5 to $2.0 \mathrm{~mm} / \mathrm{yr}$ during most the 20th century. However, such high values lead to serious misfit with geodesic observations. More recently, as reported by Mitrovica et al. (2015), the Fifth Assessment Report of the IPCC (2014) estimates the sea level changes over the period 1900-1990 to come from three contributions: $0.7( \pm 0.1) \mathrm{mm} / \mathrm{yr}$ from glaciers (including those at the periphery of the Greenland Ice Sheet), $0.4( \pm 0.1)$ $\mathrm{mm} / \mathrm{yr}$ from thermal expansion, and $-0.11( \pm 0.05) \mathrm{mm} / \mathrm{yr}$ from anthropogenic storage of water on land (we note that these values are concordant, but slightly higher than those of the 2001 IPCC report for the same period). This was in good agreement with the results by Hay et al. (2015) who estimate that over the period 1900-1990, before the beginning of a rapid general melting, the increase of the sea level, including the thermal dilatation (about $0.4 \mathrm{~mm} / \mathrm{yr}$ ), amounted to $1.2( \pm 0.2) \mathrm{mm} / \mathrm{yr}$, without significant contributions from the polar icefields over the period considered. If we adopt the correspondence established by Munk (2002) for polar regions, the eustatic change would imply an increase of the LOD of about 0.7 to $0.8 \mathrm{~ms}$ per century. However, the rise of 0.7 to $0.8 \mathrm{~mm} / \mathrm{yr}$ originates from mountain glaciers (in general at relatively high latitudes and on the coasts of Greenland), so that the variation of the LOD due to ice melting and change of the sea level should be somehow smaller then 0.7 :

$\dot{\tau}_{\text {ice }+ \text { sea }}<0.7 \mathrm{~ms} / \mathrm{cy}$.
Fortunately, there is another possible source of information. Satellite Laser Ranging (SLR) from low orbit satellite data provides information on the variation of the Earth's moment of inertia and oblateness. The Lageos I satellite range data firstly revealed significant changes of the degree -2 zonal harmonic $J_{2}$ in the development of the gravitational geopotential in spherical harmonics (Yoder et al. 1983), sensitive to the oblateness and moment of inertia. One has the following relation between the change of $J_{2}$ and $(\dot{\Omega} / \Omega)$ (Mitrovica et al. 2015),

$\dot{J}_{2}\left[10^{-11} \cdot \mathrm{yr}^{-1}\right] \simeq 2 \frac{\dot{\Omega}}{\Omega}\left[10^{-11} \cdot \mathrm{yr}^{-1}\right]$.

A change of $-1 \mathrm{~ms}$ of the LOD corresponds to the following value for $(\dot{\Omega} / \Omega)$,

$$
\Delta \mathrm{LOD} \text { of }-1 \mathrm{~ms} \quad \Longleftrightarrow \quad 11.6 \cdot 10^{-11} \mathrm{yr}^{-1} \text { for }(\dot{\Omega} / \Omega) \text {. }
$$

The result for $5.5 \mathrm{yr}$ of SLR was $\dot{J}_{2}=3 \cdot 10^{-11} \mathrm{yr}^{-1}$, it corresponds according to the above relations to a change of the LOD of $-0.51 \mathrm{~ms} / \mathrm{cy}$. For 10 year SLR from Lageos I between 1980 and 1989 , a value of $\dot{J}_{2}=2.6 \cdot 10^{-11} \mathrm{yr}^{-1}$ was found by Nerem et al. (1993), corresponding to a change of the LOD of $-0.45 \mathrm{~ms} / \mathrm{cy}$. Other results were obtained from SLR from 8 satellites from 1976 to 1995 by Cheng et al. (1997), who found $\dot{J}_{2}=2.7( \pm 0.4) \cdot 10^{-11} \mathrm{yr}^{-1} \mathrm{im}$ plying a LOD variation of $-0.47 \mathrm{~ms} / \mathrm{cy}$. More recent data of satellite laser ranging by Cheng et al. (2013) even allowed them to measure the second derivative of the $J_{2}$ parameter. However, calculation of the second derivative from inexact data is an ill-conditioned problem which is numerically very unstable. For the period 1976-1995, they find a value of $\dot{J}_{2}=2.8( \pm 0.3) \cdot 10^{-11} \mathrm{yr}^{-1}$ implying a LOD variation $\dot{\tau}_{\mathrm{J} 2}=-0.48( \pm 0.05) \mathrm{ms} / \mathrm{cy}$. The convergence of all these values is excellent. In view of the acceleration of the motion, it seems preferable to keep the slightly lower value of $-0.45 \mathrm{~ms} / \mathrm{cy}$ from 1980 to 1989 .

The variation of $\dot{J}_{2}$ implies a change of the LOD, here called $\dot{\tau}_{\mathrm{J} 2}$, which accumulates the effects on the moment of inertia due to changes of the sea level resulting from ice melting and those due to the global isostatic adjustment (GIA),

$\dot{\tau}_{\mathrm{J} 2}=\dot{\tau}_{\mathrm{GIA}}+\dot{\tau}_{\text {ice }+ \text { sea }}$.

In Sect. 4.3 below, we show that the GIA decreases the moment of inertia, implying a negative value of $\dot{\tau}_{\mathrm{GIA}}$. The value obtained by Mitrovica et al. (2015) from new Earth's model with a high viscosity in the lower mantle is $\dot{\tau}_{\text {GIA }}=$ $-1.07 \mathrm{~ms} / \mathrm{cy}$. Thus, this result implies,

$\dot{\tau}_{\text {ice }+ \text { sea }}=(+1.07-0.45) \mathrm{ms} / \mathrm{cy}=0.62 \mathrm{~ms} / \mathrm{cy}$. 
Table 1 The various contributions to the change of the LOD with and without cosmological effect on the lunar recession. The cosmological effect of $2.75 \mathrm{~cm} / \mathrm{yr}$ corresponds to the Hubble expansion with $H_{0}=70$ $\mathrm{km} \mathrm{s}^{-1} \mathrm{Mpc}^{-1}$, if acting locally in the Earth-Moon system. Column 1 gives the lunar recession considered of cosmological origin. Column 2 gives the tidal effect corresponding to the part of the lunar recession which is not of cosmological origin in ms/cy. In column 3 , only the semi-diurnal solar tide $\mathrm{S} 2$ is acting on long timescales. Columns 4-6 give the values in ms/cy of other effects contributing to the LOD over centuries. Column 7 gives $\dot{\tau}_{\mathrm{mtl}}$ which is the total change of the LOD as expressed by Eq. (13). The data in the last column have to be compared to the observed change of the LOD, either to $1.09 \mathrm{~ms} / \mathrm{cy}$ as determined from lunar occultations between 1680 and 2020 (Sect. 5.3) or to $1.78 \mathrm{~ms} / \mathrm{cy}$ from eclipses in the Antiquity and Middle Ages (Sect. 5.1)

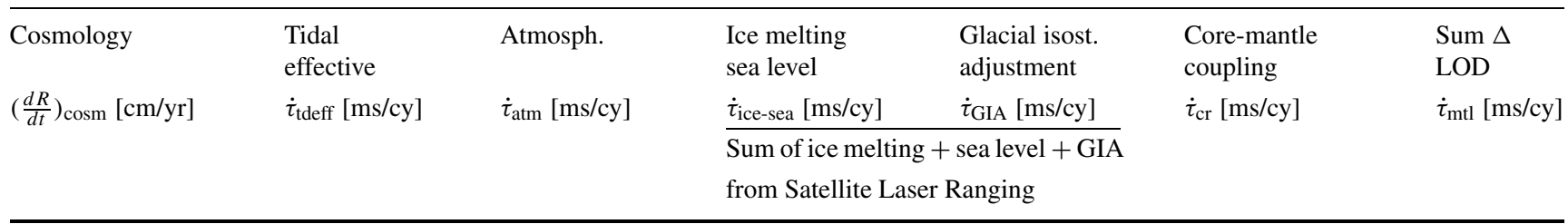

No cosmol. effect:

$0 \mathrm{~cm} / \mathrm{yr}$

2.395

$-0.05( \pm 0.02)$

$0.62-1.07$

$0.54( \pm 0.4)$

$2.44( \pm 0.4)$

$-0.45( \pm 0.05)$

With cosmol. effect:

$2.75 \mathrm{~cm} / \mathrm{yr}$

0.68

$-0.05( \pm 0.02)$

0.62

$-1.07$

$0.54( \pm 0.4)$

$0.72( \pm 0.4)$

$-0.45( \pm 0.05)$

This result is consistent with the estimate we have in expression (14) and we apply this estimate in Table 1.

Summary on ice melting and sea level effects on the LOD: The value of 0.7 to $0.8 \mathrm{~mm} / \mathrm{yr}$ of eustatic rise of sea level for the period 1900-1990 is well supported by most authors and the IPCC. As a considerable deglaciation over the whole world followed the Small Ice Age of the 17th century, we consider this value as a representative mean for the last three centuries. The exact value has little importance, since this is the sum $\dot{\tau}_{\mathrm{J} 2}=\dot{\tau}_{\mathrm{GIA}}+\dot{\tau}_{\text {ice }+ \text { sea }}$ which intervenes in the global estimate of the LOD and the error on the sum $\dot{J}_{2}$ is rather small, of about $10 \%$. As derived above, a value of $+0.62 \mathrm{~ms} / \mathrm{cy}$ may be estimated for the change of the LOD due to ice melting and change of the sea level.

\subsection{The glacial isostatic adjustment (GIA)}

Let us now turn to the "global isostatic adjustment" (GIA), also previously called "viscous rebound". At the end of the ice age $20000 \mathrm{yr}$ ago, the sea level was about $125 \mathrm{~m}$ lower than today, the difference was kept in enormous ice packs (up to $4 \mathrm{~km}$ thick) covering high latitude regions, see Peltier (1999) and Munk (2002). About $7000 \mathrm{yr}$ ago, the sea level was only $2 \mathrm{~m}$ lower and since about $4000 \mathrm{yr}$ the deglaciation is almost complete (Munk 2002).

The disappearance of the ice load is followed by a global isostatic adjustment. It is characterized by a flow, both vertically and horizontally, of viscous matter from the Earth's interior towards regions previously glaciated and mainly located at high latitudes. The rebound reaches still today up to $1.1 \mathrm{~cm} / \mathrm{yr}$ in the SE of the Hudson Bay (Peltier 1999). Such displacements are producing a decrease of the Earth's moment of inertia, the rebound reducing the Earth's oblateness. The equations of the radial and tangential displacements as a function of latitude, longitude and time are expressed as a function of the ice load and its spatial and time variations, see review by Peltier (2004). The isostatic adjustment is also a function of the viscosity parameter varying with depth, of the terms in the development of the Earth's potential and their time dependence, of the topography, etc. Different successive models (particularly VM1 and VM2) of viscosity parameters have been developed over the years. The models have a great sensitivity to the viscosity down to a depth of 2000-3000 km in the lower mantle. The influence of the Earth rotation on the GIA has been emphasized by Mitrovica et al. (2001), who also performed models of the rebound vectors over the world.

The models by Peltier have been tested by comparing the coastal changes of the sea level over thousands of years in various locations differently affected by the GIA (Peltier 2004). The relative sea level change (with account of the rebound) reaches up to $250 \mathrm{~m}$ in the South East of the Hudson Bay (with respect to $9000 \mathrm{yr}$ ago), a similar value is observed in the Gulf of Bothnia. These extreme changes have to be compared to the average sea level, which $9000 \mathrm{yr}$ ago was about $12 \mathrm{~m}$ lower than at present, see Fig. 1 by Munk (2002). The characteristic relaxation time is of $3400 \mathrm{yr}$ in the SE of the Hudson Bay and $4200 \mathrm{yr}$ in the Gulf of Bothnia (Peltier 2004). The timescales of the order of several thousands of years are such that the contemporary deglaciation has no effect yet (Sung-Ho et al. 2016), while the presently observed isostatic adjustment only results from the melting of the great glaciers of the Pleistocene. By inversion tech- 
nique, such observations in different coastal regions provide observational constraints on the viscoelastic inner structure of the Earth and on the spatial and time evolution of the glaciations. This form the basis of the ICE-5G (VM2) reference model (Peltier 2004).

For long, there were several rather converging estimates of the GIA contributions to the change of the LOD. For the secular trend in the LOD, Mitrovica and Forte (1997) found an acceleration of the Earth rotation amounting to $-0.5 \mathrm{~ms} / \mathrm{cy}$ on the basis of a model fitting the GIA decay times and the surface gravity anomalies. The effect of GIA on the LOD was estimated to be consistent with $-0.47 \mathrm{~ms} / \mathrm{cy}$ by Johnston and Lambeck (1999) from constraints on the sizes, locations and timing of deglaciation of the Late Pleistocene ice sheets and current changes in polar ice caps. Munk (2002), in a critical discussion of the various terms intervening in the LOD, is supporting a value of $-0.6 \mathrm{~ms} / \mathrm{cy}$, on the basis of a geodynamic model (Peltier 1998, 1999), applied successfully to tide gauges over the world and which also gives consistency results for the polar wander. At that time, Munk considered that this value could account for the difference between the tidal effect of $2.39 \mathrm{~ms} / \mathrm{cy}$ and the historic estimate of $1.78 \mathrm{~ms} / \mathrm{cy}$ given by Stephenson et al. (2016), however the situation appears more complex.

The effects of the GIA very much depend, as discussed above, on the viscosity between the basis of the lithosphere and the core-mantle limit (Peltier 2004). With a low viscosity, the timescale of the adjustment is short and the model rapidly recovers its equilibrium. The available observations do not seem to support such a situation. With a very high viscosity, the changes are damped. The maximum effect corresponds to a mean viscosity. The Earth's models with standard viscosity (VM1, VM2) give a rather weak GIA effect of about $-0.6 \mathrm{~ms} / \mathrm{cy}$, as that discussed above. The so-called MF viscosity model by Mitrovica et al. (2015) is different, with a much higher viscosity in the lower mantle. Starting at the surface with a standard viscosity, the MF model has a fast growing viscosity with depth, reaching a value of $2 \cdot 10^{22} \mathrm{Pas}$ in the lower mantle, 6.3 times larger than in standard VM2 models. Such a high viscosity in the lower mantle also corresponds to the finding by Nakada and Okuno (2003) and Mitrovica and Forte (2004). These differences in the model viscosity strongly change the amplitude and the time response, leading to a large GIA signal as well as a consistent polar wander. These results have led (Mitrovica et al. 2015) to considerably revise the estimate of the GIA effect. Their Fig. 3B gives a GIA contribution of $(\dot{\Omega} / \Omega)=12.4 \cdot 10^{-11} \mathrm{yr}^{-1}$. The acceleration of the angular velocity obtained with MF model corresponds to a change of the LOD of $-1.07 \mathrm{~ms} / \mathrm{cy}$, according to the expressions given in Sect. 4.2. The vector expressing the polar wander obtained from the MF model is also in agreement with observations. This new value of $\dot{\tau}_{\mathrm{GIA}}$ is very different from previous estimates. Since $\dot{\tau}_{\mathrm{J} 2}=\dot{\tau}_{\mathrm{GIA}}+\dot{\tau}_{\text {ice }}+$ sea is equal to $-0.45 \mathrm{~ms} / \mathrm{cy}$ from SLR observations, the above value allowed us in the last Section to ascertain the LOD effects of ice melting and sea level changes. On the whole, the geodesic SLR data provide the most useful internal consistency check.

Summary on GIA effects on the LOD: Most authors were supporting a moderate value of $\dot{\tau}_{\mathrm{GIA}}=-0.6 \mathrm{~ms} / \mathrm{cy}$. This value of the GIA effect was difficult to reconcile with the geodesic results from SLR measurements. A new study by Mitrovica et al. (2015) with a much higher viscosity in the lower mantle leads to a different value $\dot{\tau}_{\mathrm{GIA}}=-1.07 \mathrm{~ms} / \mathrm{cy}$. When due account is given to the ice melting and change of the sea level, a good agreement with SLR geodetic observations and polar wander is obtained.

\subsection{Effects of the inner Earth's structure and core-mantle coupling}

Differential motions in or near the Earth's core may be due to convective motions and/or differential rotation. Such motions are usually thought to be the source of the Earth's magnetic field. These motions may also operate some transfer of angular momentum between the core and the mantle and have an effect on the LOD (Hide 1989; Dumberry and Bloxham 2006; Souriau and Calvet 2015). The inner solid core of the Earth with a radius of $1220 \mathrm{~km}$ is surrounded by a liquid outer core of radius of about $3500 \mathrm{~km}$, below the solid mantle (Souriau and Calvet 2015).

The question of a high differential rotation between the inner and outer core has been much debated. On one side, there were suggestions of fast differential rotation of the inner core ( 2 or 3 degrees per year), on the other side there were more conservative studies suggesting a differential rotation at least ten times smaller. For example, an improved analysis of waves emitted by three earthquakes in South Atlantic traveling through the core and analyzed with 37 seismometers in Alaska supported an inner core spinning only slightly faster ( 0.2 to 0.3 degrees per year) than the outer core (Creager 1997). The more recent estimates from seismic observations suggest a low value of about 0.1 or less than 0.2 degree per year, the core turning slightly faster than the mantle towards the East, see Deuss (2014) and Souriau and Calvet (2015) for reviews. A recent re-analysis of observations by the full Large Aperture Seismic Array of seismic waves back scattered by the inner core for two soviet nuclear tests of 1971 and 1974 show time shifts supporting a differential rotation of 0.07 degree/yr, much lower than current estimates (Vidale 2019). Tsuboi and Butler (2020) have used antipodal earthquakes and stations to analyse the time shifts of wave propagation near the core. They conclude to a differential rotation of the inner core with respect to the 
crust-mantle of 0.05 degree/yr, smaller than past studies, but in better agreement with the results of Vidale (2019).

Munk and MacDonald (1960) recognized the role of core-mantle coupling as a source of the large decadal LOD variations, on the basis of their amplitudes and timescales (we have seen in Sect. 4.1 that the exchanges with the atmosphere necessarily have a limited amplitude). They were followed by most geophysicists who "supposed that irregular 'decade' fluctuations in the LOD of several ms must be manifestations of angular momentum exchange between the core and mantle" (Hide 1989; Buffett 2015). There are, however, a few rare discordant voices, for example Sidorenkov (2016) gave arguments in favor of an origin in the drift between the lithosphere $(\sim 60 \mathrm{~km})$ and the asthenosphere $(\sim 700 \mathrm{~km})$. The 'decade' fluctuations are remarkably illustrated by the large peaks in the variations of the LOD based on lunar occultations shown by McCarthy and Babcock (1986) and Stephenson et al. (2016), as illustrated in the present Figs. 2 and 5. We first see a steep decrease of the LOD starting in 1860 and reaching a narrow minimum with $-4 \mathrm{~ms}$ around 1868. The full recovery occurred around 1890 and was followed by a strong increase of the LOD with a broad maximum of about $+4 \mathrm{~ms}$ from 1901 to 1912 until recovery around 1932. There is unfortunately no indications available on the possible cycles of such decadal oscillations.

Most remarkably, the fluid motions at the top of the core can be deduced from the observations of the variations of the magnetic field at the surface of the Earth, see review by Gross (2009) where many specific references can also be found. A number of authors have studied the possible properties of the core magnetic field and the following picture has emerged:

- The core may be considered as a perfect conductor so that the field is frozen in it and is confined at the surface of the core by advection;

- The mantle is an insulator, so that no creation or destruction of the field occurs through it and the spatial behaviour through the mantle is predictable;

- The large scale flow is geostrophic, i.e. horizontally there is a balance between the pressure gradient and the Coriolis force;

- The flow is essentially steady and has no radial component.

The first successful model able to account for the decadal LOD variations was developed by Jault et al. (1988). They demonstrated that the axisymmetric components of the rotating flow in the core are equivalently described by the relative motions of concentric cylinders rotating around the rotation axis of the core. Jault et al. were able to derive the effects of the core motions on the LOD from the variations of the field at the surface of the core, which were themselves obtained from the observations of the variations of the magnetic field at the surface of the Earth. This finding was the opening of the connection between the observed variations of the surface magnetic field and the changes of the LOD, a research line with numerous papers, see reviews by Herring (2007), Olson (2007) and Buffett (2015). A zonal rotation of the fluid is bearing the magnetic field of the core, which is symmetric with respect to the equator and mimic the westward observed surface drift with a latitudinal dependence. A timescale of about 10 years characterizes this differential rotation. Jackson et al. (1993) determined the variations of the fluid velocities at the core-mantle boundary from data on the Earth's magnetic field from the years 1840-1990. They derived the variations of the rotation of the mantle and could compare them with observations. Several comparisons between the observed decadal LOD variations and the predictions of different models based on the observed surface variations of the magnetic field have been made, see for example Ponsar et al. (2003) and Gross (2009). The agreement, although not perfect, is good enough to validate this remarkable connection between what happens at the surface of the Earth and in its deep core.

However, the way by which there is some physical transfer of angular momentum from the core to the mantle is still unclear. The basic dynamics of Earth's core and the various mechanisms of core-mantle coupling have been reviewed and analysed by several authors, for example by Jault (2003), Ponsar et al. (2003), Gross (2009), Roberts and Aurnou (2012) and Glane and Buffett (2018). Many articles and books have been devoted to the subject since fifty years, we briefly try to report on the main emerging conclusions. The different possible coupling processes are: convection, viscosity, magnetic field, topography and gravity. Viscous torques appear insufficient, as well as convection which does not generate sufficient density anomalies.

The electromagnetic coupling would be produced by the magnetic field of the core interacting with electric currents induced by the varying magnetic field in the lower mantle, which may still be weakly conducting. This mechanism can clearly make the necessary coupling of the mantle for producing the decadal observed change of the LOD (Holme 1998; Ponsar et al. 2003). However, its efficiency and thus the validity of this explanation depends on the unknown conductivity, as firstly emphasized by Jault (2003), see also Gross (2009). Recently Kuang et al. (2018) showed that a different field geometry in the outer core could make the coupling much stronger and succeed in accounting for the polar wander. About topography, the same kind of uncertain conclusion is emphasized by Jault (2003) and Gross (2009). There, the irregularities of the core-mantle boundary are "pushed" by the pressure of the liquid core and a torque is exerted on the mantle. The size of the effect depends on the unknown amplitudes of the bumps on the boundary, so that topography is a valid possibility. Another mechanism is gravity, it occurs if there are density inhomogeneities both 


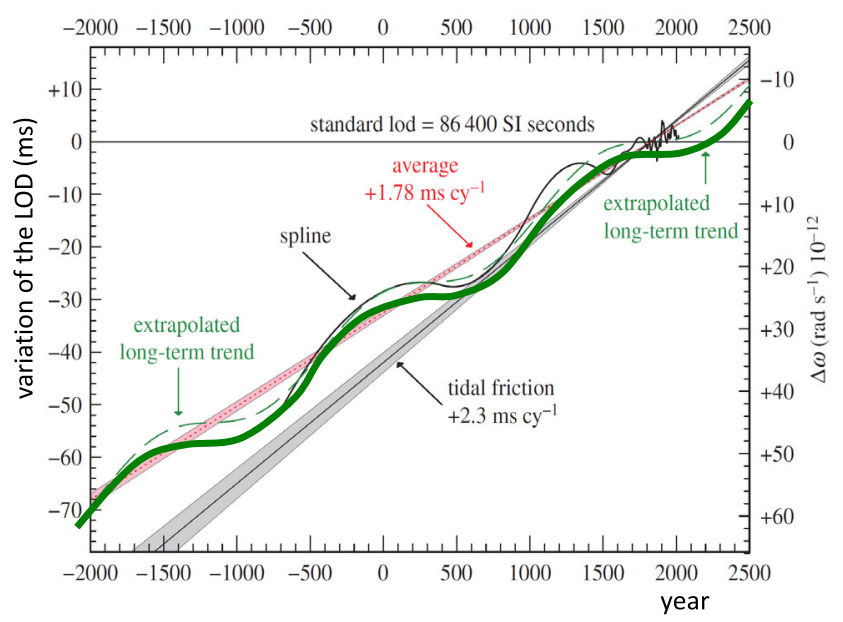

Fig. 3 Schematic representation of the change of the LOD over -2000 to 2500 , in ms on the left axis and in angle on the right axis. The pink curve represents the observed average change of $1.78( \pm 0.03) \mathrm{ms}$ per century according to astronomical data of solar and lunar eclipses. The grey line shows the tidal change of $2.3( \pm 0.1) \mathrm{ms}$ per century from the assumed tidal friction. The undulated line (reinforced here) shows the extrapolated long-term trend. From Fig. 18 by Stephenson et al. (2016). The data shows almost zero slope near year 2000 as seen in Fig. 1

in the fluid core and in the mantle. Then, the local differences of gravity can exert a torque on the mantle. Here also, the size of the inhomogeneities, which determine the gravity coupling, is unknown. Alike the other processes, this one could transmit the core variations to the LOD, but it is also resting on uncertain parameters.

The gravitational core-mantle coupling due to random density inhomogeneities in the liquid outer core and in the mantle has been advocated by Rubincam (2003) to be the source of the long-term $1500 \mathrm{yr}$ oscillations found by Stephenson et al. (2016) and illustrated in Fig. 3. A timescale of about $1000 \mathrm{yr}$ corresponds to a (rough) estimate of the dissipation time of the inhomogeneities. It is most likely that this process resting on gravity interactions of random and evanescent bubbles with limited density differences has a longer timescale than the other ones and could work for the $1500 \mathrm{yr}$ oscillation rather than for the decadal variations. This process could, according to $\mathrm{Ru}-$ bincam (2003), also account for the westward drift of the magnetic field by 0.2 degree/yr, while it appears to have a negligible incidence on the polar wander. However, the effects on the LOD are uncertain.

There are also many paleomagnetic data collected in lake sediments and old lava flows, which capture crustal features of the magnetic field. The records go back to about $7000 \mathrm{yr}$ before present, see for example Korte and Constable (2005). These data lead to some modifications of the geometry and evolution of the surface magnetic field, which shows some periodic eastward and westward motions of magnetic patterns.
Dumberry and Bloxham (2006) studied with archaeomagnetic field models the long-term behaviour of the physical effects in the core-mantle coupling and found significant differences in the flows with respect to the case of the short-term decadal variations. In particular, in the long-term variations there are shears in the direction of the rotation axis, with a transport of angular momentum mainly parallel to this direction. At decadal timescale, "vertical" oscillations dominate the transport, which transmits the angular momentum perpendicularly to the rotation axis. The similarity of the period of these archaeomagnetic secular variations to the 1500 yr oscillations of the LOD suggests, according to Dumberry and Bloxham (2006), that both variations of the magnetic field and the LOD originate from angular momentum exchange between the Earth's core and the mantle. By inversion techniques similar to those applied for the decadal variations of the LOD, these authors obtained data on the azimuthal magnetic flows at the surface of the core over the period between 1000 BC and 1820 AD. Thus, Dumberry and Bloxham (2006) could calculate the exchange of the angular momentum between the core and the mantle and found variations at millenial-scale, consistent with the LOD variations, thus confirming the above suggestion. As pointed out by Mitrovica et al. (2015), "This prediction suggests a mean increase in Earth's rotation period over the past 2500 years as a result of core-mantle coupling". To estimate this increase, with account of the oscillatory signal, Mitrovica et al. (2015) performed calculations of the cumulative $\Delta T$ for three different time series approximating the change of rotation period. The average $\Delta T$ curve vs. time they obtained has a certain thickness, representing the uncertainties due to the different approximations. Globally, from the curve obtained by Mitrovica et al. (2015) in their Fig. 3 A, we estimate that the secular change of the core-mantle coupling is about the half of the shift due to the GIA, but with an opposite sign, this gives a value of $+0.54 \mathrm{~ms} / \mathrm{cy}$, with an uncertainty of about $\pm 0.4 \mathrm{~ms} / \mathrm{cy}$ for this value, if we account for the various approximations performed by Mitrovica et al. Among the various terms in Eq. (13), this is the most uncertain one, as it is based on only one model derivation and contrary to the case of the GIA, there is no SLR measurements permitting a check of consistency.

We note that the movements of the Earth's crust, the continental drifts, the plate subduction and the Earth's cooling have much longer timescales, of the order of million years. As to the effects of the inner structure of the Moon on the relation between the LOD and the lunar recession, they are much smaller than those considered above. The consequences of a possible presence of a liquid, molten, or solid core in the Moon have been studied by Williams et al. (2001), Shuanggen (2014) and the possible internal exchanges of angular momentum appear to have a negligible effect. 
Table 2 The different estimates of the secular increase of the LOD

\begin{tabular}{lll}
\hline Source and period of observations & $\begin{array}{l}\text { Change of the LOD } \\
\mathrm{ms} / \mathrm{cy}\end{array}$ & References \\
\hline Ancient eclipses 700 BC - 1600 AD & 1.40 & Stephenson and Morrison (1984) \\
Comparison of ephemeris 1875-1970 & 2.49 & Goldstein (1985) \\
Lunar occultations 1656-1986 & 0.73 & McCarthy and Babcock (1986) \\
Ancient eclipses 700 BC - 1600 AD & 1.70 & Stephenson and Morrison (1995) \\
Combined data 1832.5 -1997.5 & - & Gross (2002) \\
Lunar occultations over last 350 yr & 0.90 & Sidorenkov (2005) \\
Ancient eclipses 720 BC - 1600 AD & 1.78 & Stephenson et al. (2016) \\
Paleontology $~ 1$ Gyr & 1.64 & Deines and Williams (2016) \\
Sea level change since 3000 yr & 0.00 & Hay et al. (2016) \\
Babylonian obs. 419 BC -89 BC & - & Gonzales (2018) \\
Lunar occultations + IERS $1680-2020$ & 1.09 & This work
\end{tabular}

Summary on core-mantle coupling effects on the LOD: It appears established that the core-mantle coupling produces variations of the Earth's magnetic field and of the LOD. On the basis of archaeomagnetic field models, Dumberry and Bloxham (2006) have studied the exchange of angular momentum between the Earth's core and the mantle. Following Mitrovica et al. (2015), who support an increase in Earth's rotation period, we consider a value $\dot{\tau}_{\mathrm{cr}}=0.54 \mathrm{~ms} / \mathrm{cy}$, derived from their Fig. $3 \mathrm{~A}$ on the basis of the models by Dumberry and Bloxham (2006). We estimate that this value is much more uncertain than for the other atmospheric and geophysical effects considered, which could be checked by SLR measurements.

Table 1 summarizes the change rates of the LOD for the various effects examined. The case without a cosmological contribution is presented in block 1 , with lines 1 and 2 . In block 2, with lines 3 and 4, it is assumed that the Hubble expansion with $H_{0}=70 \mathrm{~km} \mathrm{~s}^{-1} \mathrm{Mpc}^{-1}$ is also present in the Earth-Moon system. This corresponds to a lunar recession of $2.75 \mathrm{~cm} / \mathrm{yr}$. The difference with observed lunar recession of $3.83 \mathrm{~cm} / \mathrm{yr}$ is $(d R / d t)_{\text {tdeff }}=1.08 \mathrm{~cm} / \mathrm{yr}$, thus assumed to originate only from the tidal effect, see Eq. (6). The corresponding tidal slowing down of the Earth rotation would thus be $0.68 \mathrm{~ms} / \mathrm{cy}$ instead of $2.395 \mathrm{~ms} / \mathrm{cy}$. Interestingly enough, the sum of the various positive and negative contributions (13) other than the tidal one appears to be rather small of about $+0.04 \mathrm{~ms} / \mathrm{cy}$. When all contributions to the LOD are accounted for, one obtains the results in the last column of Table 1 . In the case without a cosmological effect, a slowing down of $2.34 \mathrm{~ms} / \mathrm{cy}$ is obtained, while it is $0.62 \mathrm{~ms} / \mathrm{cy}$ if the Hubble expansion is present. These values are to be compared to observations.

\section{Survey of data sources on the variations of the LOD}

There are different sources of information on the changes of the LOD:

- The study of the ancient solar and lunar eclipses over the last $2700 \mathrm{yr}$;

- The analysis of lunar occultations of bright stars and planets, with precise times, since the middle of the $17^{\text {th }}$ century;

- The variations of the sea level;

- The precise geodetic measurements of the Earth rotation since 1962 with the IERS.

\subsection{Ancient observations of eclipses}

The study of ancient lunar and solar eclipses offers a powerful test, since large cumulative shifts $\Delta T$ may result between the time UT dependent on Earth rotation and TT based on constant days of 86400 SI seconds. For example, over an interval of $2000 \mathrm{yr}$, the shift of the timescale is of the order of $18000 \mathrm{~s}$. Such differences correspond to displacements of the predicted geographic locations of the eclipse visibility over large distances, up to a few thousand $\mathrm{km}$. Stephenson and Morrison (1984) determined from eclipse data from 700 $\mathrm{BC}$ to $1980 \mathrm{AD}$ a change of the LOD by $1.4 \cdot 10^{-5} \mathrm{~s} \cdot \mathrm{yr}^{-1}$. Later (Stephenson and Morrison 1995) corrected their previous value and gave $1.70 \cdot 10^{-5} \mathrm{~s} \cdot \mathrm{yr}^{-1}$. A further study by Stephenson et al. (2016) lead to $1.78 \mathrm{~ms} / \mathrm{cy}$ from the data prior to AD 1600, a work often used as a comparison basis for the LLR results. This value is much lower than the value of $2.395 \mathrm{~ms} / \mathrm{cy}$ (Williams et al. 2016; Williams and Boggs 2016) for a tidal interaction producing the observed $3.83 \mathrm{~cm} / \mathrm{yr}$ lunar recession, see also Stephenson et al. (2020) who consider the difference as significant. 
In the works of 1995 and 2016, Stephenson et al. found an undulation with an amplitude of about $4 \mathrm{~ms}$ around the mean with a period of about $1500 \mathrm{yr}$, so that at some epochs the centennial increase of the LOD is higher than the mean and lower at other epochs, see Fig. 3 derived from Fig. 18 by Stephenson et al. (2016). For example, the average increase would be lower between about AD 1600 and 2100 . This long-term undulation has often been a source of stimulation and a comparison basis for the studies of the coremantle coupling, as seen in the previous Section. However, as noted by Stephenson et al. (2016) about the $1500 \mathrm{yr}$ undulation, "this is less reliably established because it is critically dependent on relatively few observations."

Dalmau (1997) criticized the results by Stephenson and Morrison (1995), in particular the treatment of the Arabic records and the statistical method employed: "the authors listed above have not evaluated the Arabic records with the sufficient care. They have taken the numerical data from the sources without considering the context. The statistical method used by them meets by no means the requirements of the subject." Hay et al. (2016) have pointed out that the inferred departure from a quadratic relation proposed by Stephenson et al. is driven by a relatively small number (11) of solar eclipses untimed observations (without indications of the eclipse duration). Soma and Tanikawa (2016) have used two ancient Japanese occultations of Venus in AD 503 and of Saturn in AD 513 to test the secular change of the LOD. For both observations, they found a $\Delta T$ much smaller, with a difference between about $400 \mathrm{~s}$ and $2700 \mathrm{~s}$, than the smoothed value of Stephenson (1997). This difference is supported by the data from solar eclipses in AD 516 and 522 . Their conclusion was that in the beginning of the sixth century $\mathrm{AD}$, the $\Delta T$ values were well below the smoothed trend obtained by Stephenson (1997). Another eclipse of AD 454 showed a higher $\Delta T$ at that time, suggesting fast variations. The Babylonian occultations and appulses between 80 and 419 BC were studied by Gonzales (2018), who suggested a small revised version of the quadratic fit with respect to Stephenson et al. (2016). Thus, despite their high interest the ancient observations contain sources of uncertainties, which may affect the conclusions. A new independent study would be most useful in the context.

\subsection{Previous studies from lunar occultations}

From AD 1623 up to 2015 there were $478^{\prime} 843$ observations of timed occultations of stars by the Moon collected by Herald and Gault (2012) in CDS at Strasbourg, who provided a most reliable sample. Before the middle of the $17^{\text {th }}$ century, the timing of the occultations was often inaccurate and the observations show a large scatter. McCarthy and Babcock (1986) have made a detailed study of the evolution of the LOD for the period of AD 1656 to 1986. The observations come from four different sources, one of them is McCarthy himself observing at the US Naval Observatory photographic zenith tube. All data reduced to the same timescale, with the values of the LOD and their errors, are given in the paper. Some light smoothing has been applied, particularly for the data before 1820 . Over the whole period, McCarthy and Babcock (1986) found a mean decrease of the angular velocity of the Earth of

$$
\frac{\dot{\Omega}}{\Omega}=-8.433( \pm 0.207) \cdot 10^{-11} \mathrm{yr}^{-1} .
$$

Converted in term of the LOD, this braking corresponds to a change of $+0.73( \pm 0.018) \mathrm{ms} / \mathrm{cy}$. The curve obtained by McCarthy and Babcock (1986) is illustrated in Fig. 2, where the authors have shown only the deviations around the mean value given by the above equation. We see that over an interval of $330 \mathrm{yr}$ the mean of the data are very well represented by a horizontal line. One could wonder whether the decline appearing near the right end of the plot (year 1986) is further going on and supporting the general flat curve. This is the case as illustrated by Fig. 1 of the IERS data, which shows that the decline after 1986 is going on with further oscillations around relatively low LOD values.

The McCarthy and Babcock (1986) data over the last 3.3 centuries were supporting a secular change of the LOD about 2.4 times smaller than the values from antique observations. Commenting on this difference, McCarthy and Babcock (1986) noted the possibility that errors in the ancient observations have led to systematic errors by Stephenson and Morrison. Another possibility is that the secular lengthening since 1656 is different from the overall trend from 700 $\mathrm{BC}$ to $1600 \mathrm{AD}$. However, it is surprising that this large difference in the LOD evolution should coincide with the appearance of accurate time determinations and telescopic observations.

From astronomical telescopic observations over the last $350 \mathrm{yr}$, Sidorenkov (2005) found a value of $0.9 \cdot 10^{-5} \mathrm{~s} \mathrm{yr}^{-1}$ for the secular change of the LOD. This result is not very different of that by McCarthy and Babcock (1986). The value by Sidorenkov has been first used by Dumin (2005) to study the possibility of cosmological expansion in the Solar System due to Dark-Energy effects and then in further studies on local expansion (Dumin 2007, 2016). A combined LOD series spanning the years 1832-1997 has been established by Gross (2002). It is based on lunar occultations, optical astronomy, satellite laser ranging and VLBI. Over the period considered, the observations are in excellent agreement with McCarthy and Babcock (1986) and Stephenson and Morrison (1984). Table 2 collects the different estimates of the secular variation of the LOD.

The evolution of the LOD, derived from high quality lunar occultations since about 1700, is shown in Fig. 19 by Stephenson et al. (2016). This figure is given in Fig. 5 below. It confirms the relatively low change of the LOD over the 
last centuries in agreement with the results by McCarthy and Babcock (1986) and Sidorenkov (2005). Many oscillations of various characteristic times are visible in this figure, in particular the so-called decadal fluctuations with large peakto-peak amplitudes with a strong one between the years 1860 and 1935, see Sect. 4.4.

\subsection{New study of the LOD from lunar occultations}

Some time having passed since the work by McCarthy and Babcock (1986), it is useful to make another study of the LOD covering the recent centuries up to the present. We take the tabulated data of lunar occultations by Stephenson et al. (2016) from 1680 and extend them to 2020 thanks to the recent IERS values (see the IERS URL to the DataProducts/EarthOrientationData/eop.html). The thin black curve in Fig. 5 illustrates the sequence of lunar occultations given by Stephenson et al. The other thin curves show other data (see caption). In their common parts, they agree well.

A linear regression of the LOD based on data from the year 1680 to 2020 gives the following expression,

$\operatorname{LOD}[\mathrm{ms}]=0.01089 \cdot$ year $[\mathrm{AD}]-20.068$

As seen from (20), the zero of LOD is in years 1842-1843 in agreement with Fig. 3. The zero in this case corresponds to a day of about 86400 SI seconds exactly. While, the secular change of the LOD given by the above relation is:

Slope of the LOD $=1.09( \pm 0.012) \mathrm{ms} / \mathrm{cy}$,

where the $90 \%$ confidence limit is indicated. The regression line given by Eq. (20) is illustrated by the thick black line in Fig. 5. The main deviations from the mean trend in the observations shown in this figure are similar to those of Fig. 2 by McCarthy and Babcock (1986) except that unlike in Fig. 2 no adjustment for the mean value have been applied. The present result on the change of the LOD is supporting a relatively low value, relatively close to the result by McCarthy and Babcock (1986) and by Sidorenkov (2005), despite the differences in the start and the end of the periods considered.

In the calculations for (21), the tabulated data of lunar occultations by Stephenson et al. (2016) was utilized. To obtain the change in the LOD Stephenson et al. (2016) applied cubic splines fit to their data about the time change $\Delta \mathrm{T}$. Where $\Delta T=T T-U T$ is defined as the difference between the theoretically uniform time scale, which is denoted by Terrestrial Time (TT), and the (variable) rotational period of the Earth denoted by Universal Time (UT). The cubic spline fit allowed for evaluating the time derivative of $\Delta \mathrm{T}$ and thus estimating the change in the LOD, which was provided in their tabulated results. To validate the values obtained from

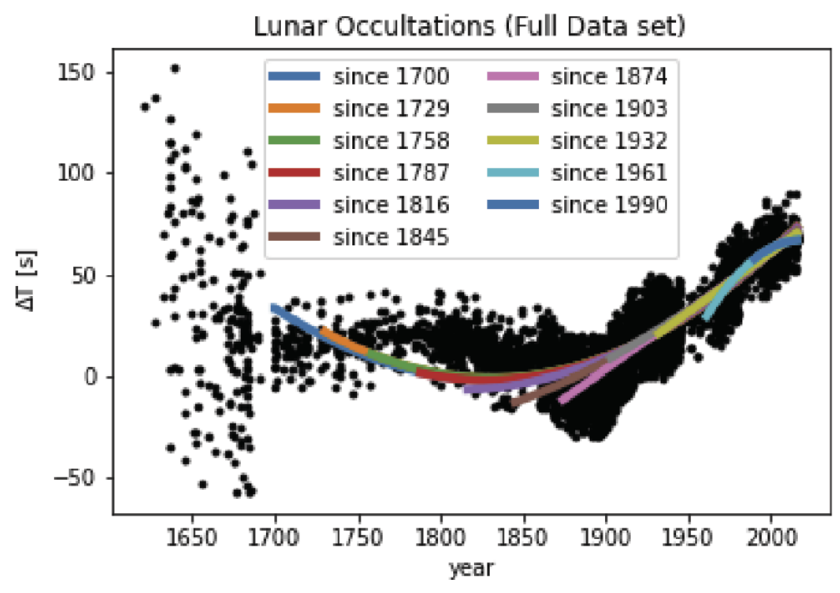

Fig. 4 Lunar Occultation data on $\Delta \mathrm{T}$ from the original data provided in the supplemental material of Stephenson et al. (2016). The corresponding quadratic polynomial fit using the records as indicated are shown

the cubic spline fit method they utilized also local polynomial regression (loess) with a quadratic smoothing parameter throughout after 1600 . The results based on the cubic spline fit and loess can be seen in the original Fig. 19 of Stephenson et al. (2016), as well as in Fig. 5 here. Stephenson et al. (2016) have also used parabolic fit to a larger data set -720 to 2015 with the unit of the second on the Ephemeris Time (ET) scale determined from the observations between 1750 and 1892 and were carefully discussed in the computation of $\Delta T$ (Stephenson et al. 2016) along with the Lunar ephemeris and various relevant observations and comparisons.

In order to identify the size of the data needed to detect the long term impact in the change of the LOD, we have used the original records by Stephenson et al. (2016) since year 2020 with backwards time increments of 29 years and have utilized a polynomial of second degree for our least square fit. The corresponding best fit curves are shown in Fig. 4. The resulting coefficients are shown in Table 3 along with the relevant slope value. As seen from the table, after 200 years of data the change of the LOD settles at the value consistent with the one computed in (21) based on the cubic spline fit tabulated results. Thus, in order for the effect to be confirmed and to become visible within the highly accurate modern IERS data, another 150 years of collection of new data may be needed.

The difference between the above results and the data from ancient eclipses between $720 \mathrm{BC}$ and $1600 \mathrm{AD}$ is quite large. Both sampling have their respective advantages and weaknesses. The eclipse data are much less accurate, but they cover a much longer period of time. The occultation data are much more accurate, but they cover a shorter period. As already pointed out by McCarthy and Babcock (1986), there are two possibilities. A) "It is possible that errors in the ancient observations have resulted in a systematic error in 
Table 3 Values of the LOD change (slope) using data sets starting since the indicated year and ending in 2020 . The slope value is given by the expression $2 \times 100 \times 365 \times\left(10^{-6} \times a_{2}\right)$. The $a_{i}$ are the coef- ficients deduced via quadratic least-squire fit to $\Delta \mathrm{T}$ as function of the Julian day. The $\chi$ is the square root of the residuals per data sample adjusted for the degrees of freedom

\begin{tabular}{|c|c|c|c|c|c|}
\hline $\begin{array}{l}\text { Record } \\
\text { since }\end{array}$ & $\begin{array}{l}\text { Slope } \\
{[\mathrm{ms} / \mathrm{cy}]}\end{array}$ & $\begin{array}{l}a_{2} \\
{\left[\mathrm{~ns} / \mathrm{day}^{2}\right]}\end{array}$ & $\begin{array}{l}a_{1} \\
{[\mathrm{~s} / \text { day }]}\end{array}$ & $\begin{array}{l}a_{0} \\
{[\mathrm{~s}]}\end{array}$ & $\chi$ \\
\hline 1700 & 1.1681 & 16.0020 & -0.0765 & 91316 & 4.8264 \\
\hline 1729 & 1.1988 & 16.4222 & -0.0785 & 93804 & 4.8108 \\
\hline 1758 & 1.1983 & 16.4147 & -0.0785 & 93759 & 4.8058 \\
\hline 1787 & 1.1651 & 15.9596 & -0.0762 & 91061 & 4.7866 \\
\hline 1816 & 1.0195 & 13.9659 & -0.0665 & 79229 & 4.6512 \\
\hline 1845 & 0.6348 & 8.6963 & -0.0408 & 47925 & 4.2468 \\
\hline 1874 & 0.0177 & 0.2426 & 0.0004 & -2362 & 3.4032 \\
\hline 1903 & 0.4243 & 5.8124 & -0.0268 & 30890 & 2.8724 \\
\hline 1932 & 0.0639 & 0.8756 & -0.0027 & 1299 & 2.3726 \\
\hline 1961 & -6.0413 & -82.7581 & 0.4070 & -500217 & 1.3003 \\
\hline 1990 & -6.1537 & -84.2970 & 0.4145 & -509459 & 1.0348 \\
\hline
\end{tabular}

the estimate of Stephenson and Morrison". This possibility could be supported by some remarks by Dalmau (1997) and Hay et al. (2016). Also, it is bit surprising that this change of LOD regime just corresponds to the epoch where timed observations started. B) The difference is real and represents a systematic change of the LOD. The $1500 \mathrm{yr}$ oscillation, if real, might be an explanation. (This $1500 \mathrm{yr}$ ondulation has been considered very seriously by geophysicists studying the core-mantle coupling, see Sect. 4.4).

In Fig. 3, we see that from $720 \mathrm{BC}$ to to $1600 \mathrm{AD}$, there are two phases where the increase rate of the LOD is high (from about $700 \mathrm{BC}$ to $100 \mathrm{BC}$, and from about $900 \mathrm{AD}$ to $1600 \mathrm{AD}$ ), while there is only one phase (from about $100 \mathrm{AD}$ to $700 \mathrm{AD}$ ) where the rate is shorter than the mean of $1.73 \mathrm{~ms} / \mathrm{cy}$. This difference is biasing the mean increase rate of the LOD by Stephenson et al. (2016) towards a high value, since the mean rate is the mean slope of the cumulative effect $\Delta T$ vs. time. We may call this the "high-average regime". The period from about 1600 AD to 2100 AD lies in the relatively "flat regime" of the undulation, where the increase rate of the LOD is lower than the mean. Thus, this could explain the difference of the results by Stephenson et al. (2016) with those of McCarthy and Babcock (1986) and ours. In this respect, we note that the period of 1970 to 2015, where the LLR observations have been made, is also fully lying in such flat regime, which may account for its peculiarity.

\subsection{The LOD from the IERS. Other tests}

The most accurate information on the contemporary variations of the LOD is provided by the International Earth Rotation Service (IERS). The studies are based on ground based observations, geodetic satellites and VLBI observations of most distant objects to ascertain the various geodesic parameters and the LOD in particular (Herring 2000). The consistency of recent data for Earth rotation and gravity field parameters obtained from Satellite Laser Ranging (SLR) has also been demonstrated (Sosnik et al. 2016). Figure 1 illustrates the LOD, the excess revolution time, from 1962 to 2020. The decadal fluctuations in this interval prevent, for the time being, any derivation of a mean trend on such a small period of time.

It is worth noticing that the IERS data shows slight speeding of the earth rotation resulting in a shorter day of about $0.0001 \mathrm{~ms} / \mathrm{yr}$, which is easily noticeable by eye inspection of the data in Fig. 1. Since the melting of glaciers at moderate latitudes and polar ice-fields has no effect anymore and actually results in the opposite sign of the LOD change, as discussed in 4.2, it is an interesting question for mathematical modeling if this numerical value of $1 \cdot 10^{-7} \mathrm{~s} \mathrm{yr}^{-1}$ could be explained by the disappearing of the mountaintop glacier ice in the tropics around the world due to the observed accelerated environmental changes (Thompson et al. 2021), or if this is due to global warming effects in the semi-diurnal atmospheric tide $\mathrm{S} 2$ which has the same-sign effect on the LOD change as discussed in Sect. 4.1.

We note that Goldstein (1985) gave a high value 2.49 . $10^{-5} \mathrm{~s} \mathrm{yr}^{-1}$ for the change of the LOD over the period 1875-1970 on the basis of comparisons between the longitudes and the mean motions of the Sun from ephemeris tables. However, the time period is too short to give a significant trend, although the order of magnitude is correct. Deines and Williams (2016) determined the average deceleration of the rotation rate of the Earth from paleontological fossils and deposits back to more than a Gyr ago. They obtain a deceleration rate equal to $96.6 \%$ of that of Stephenson and Morrison (1995), this corresponds to $92.3 \%$ of the rate 


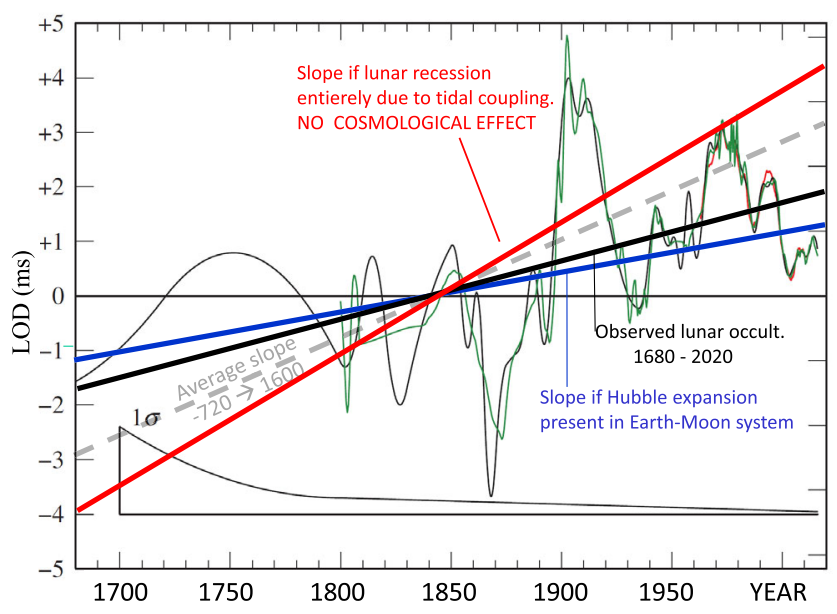

Fig. 5 The change of LOD from the lunar occultations between 1700 and 2015 according to Fig. 19 by Stephenson et al. (2016). The thin black observed curve is a spline fitting, the thin green curve after 1800 is obtained from a local moving regression (loess) and the thin red curve shows the IERS data (cf. Fig. 1). The thin lines belong to the original figure by Stephenson et al., while the thick lines are added in the present work. The thick black line is the regression line of the observations of lunar occultations obtained from the tabulated data by Stephenson et al. (2016) completed by recent IERS data. It crosses the value zero for the change of LOD in the year 1842. The other thick lines show different slopes, also attached to a LOD change of $0 \mathrm{~ms}$ in the year 1842. The thick red line indicates the slope of a change of the LOD by $2.395 \mathrm{~ms} / \mathrm{cy}$ (Williams et al. 2016), obtained if the lunar recession is entirely due to tidal coupling of the Earth-Moon system. Closely above this value would, we would have the slope of $2.44 \mathrm{~ms} / \mathrm{cy}$ corresponding to the case of no cosmological effect in the lunar recession (cf. Table 1 ). The thick grey line gives the slope of $1.78 \mathrm{~cm} / \mathrm{cy}$ obtained from ancient eclipses from 720 BC to 1600 AD by Stephenson et al. (2016). The blue line shows the slope of $0.72 \mathrm{~ms} / \mathrm{cy}$ of the LOD if the Hubble expansion participates $(2.75 \mathrm{~cm} / \mathrm{yr}$ ) to the observed lunar recession (cf. Table 1)

by Stephenson et al. (2016), which is a bit surprising since the Moon was closer to the Earth and the tidal effects larger.

Hay et al. (2016) use the sea level changes over the last $3000 \mathrm{yr}$ to infer the change of the LOD, accounting for the ice mass fluctuations, the thermal variations, the change of shore lines, the viscoelastic response of the mantle, etc. They find large differences in the change of the LOD with respect to the eclipse predictions, with a curve in sawtooth and no clear global trend. As a matter of fact, the curve they obtain for the secular variations of the LOD is compatible with $0 \mathrm{~ms} / \mathrm{cy}$ or even with a marginal acceleration of the Earth's rotation. However, we may remark that the inferences on the LOD from the evolution of the sea levels is a very convoluted test.

\section{Analysis of the LLR and LOD relations}

We now compare these various predictions and observations. The first question is which observations to consider, the ancient eclipses or the lunar occultations.

\subsection{The "high-average" and the "flat" regime}

The high mean value of the Earth's deceleration from eclipses between $720 \mathrm{BC}$ and $1600 \mathrm{AD}$, and the low value from lunar occultations between 1680 and 2020, if not due to errors, could correspond to different regimes within the $1500 \mathrm{yr}$ oscillation: the "high-average regime" and the "flat regime" respectively, as discussed previously (Sect. 5.3). This oscillation may result from angular momentum exchange between the core and the mantle, as proposed by some geophysicists and discussed in Sect. 4.4. But, the reality of the oscillation is uncertain and the same for the origin of the difference between the two regimes.

An exchange of angular momentum between the core and the mantle lets the total Earth's angular momentum unmodified, so that there is no direct effect of the exchange on the lunar recession. The change $\triangle L O D$ in the mantle only modifies insignificantly $T_{i}^{2}$ in the term $k_{i}$ so that the effect of the tidal coupling remains essentially the same.

However, the velocity of lunar recession of $3.83 \mathrm{~cm} / \mathrm{yr}$ is not an inertial velocity: if the gravitational pull exerted by the tidal deformation would suddenly be absent (or different), about one second later there would no longer be any increment (or a different one) in the Earth-Moon distance. The tidal coupling is a gravitational effect, as such it is acting at the speed of light. As the origin of the different regimes is highly uncertain (we have noted that the difference appears with the epoch of accurate observations), LLR and LOD observations in the same epoch may be an advantage.

\subsection{Comparisons with different solutions}

Figure 5 presents graphically the main results of this work. The thick black straight line represents the mean of the observations of lunar occultations + IERS data, as described by Eq. (21) with a slope of $1.09 \mathrm{~ms} / \mathrm{cy}$. Three other slopes are shown in Fig. 5, also attached in the year 1842 to a LOD change equal to zero. The first one in red color represents a change of $2.395 \mathrm{~ms} / \mathrm{cy}$, corresponding to the case where the whole observed lunar recession would be due to the tidal effect (Williams et al. 2016). This red line is steeper by a factor 2.2 than the general observed trend over $340 \mathrm{yr}$, this is quite a large difference, larger than expected from a combination of the tidal and the geophysical effects studied in Sect. 4. Thus, the low slope observed over $340 \mathrm{yr}$ makes it difficult to support that the whole lunar recession is due to tidal effect. We also see from Table 1 that if no cosmological effect is present, accounting for all corrections, we would have a slope of $2.44 \mathrm{~ms} / \mathrm{cy}$ (which would be slightly steeper than the red line). This last solution and the red line are also not in agreement with the grey line giving the slope from eclipses in the Antiquity and Middle Age, despite all the geophysical corrections applied to the LOD. Thus, there 
may still be some unknown effect accelerating the Earth rotation, or there may be another significant expansion present in the observed lunar recession, whatever data is considered.

The grey broken line in Fig. 5 represents the slope of $1.78 \mathrm{~ms} / \mathrm{cy}$ obtained with the LOD data from $720 \mathrm{BC}$ to 1600 AD by Stephenson et al. (2016). It is certainly a "highaverage" value since the period considered contains two phases of fast change, if the oscillations of the LOD are real. As noted above, it is in disagreement with both the results with and without a cosmological effect, the difference with respect to the results of the last $340 \mathrm{yr}$ being larger.

We now examine the blue slope in Fig. 5. It corresponds to the third line in Table 1 , where it is assumed that the Hubble-Lemaître expansion with $H_{0}=70 \mathrm{~km} \mathrm{~s}^{-1} \mathrm{Mpc}^{-1}$ is acting in the Solar System. This means an expansion of $2.75 \mathrm{~cm} / \mathrm{yr}$ of cosmological origin in the Earth-Moon system among the observed $3.83 \mathrm{~cm} / \mathrm{yr}$. In this case, a recession of only $1.08 \mathrm{~cm} / \mathrm{yr}$ would be due to the tidal interaction with the Earth. Wether such a low tidal interaction would be compatible with the Earth's oblatness remains an open question. The conservation of the angular momentum would thus imply a change of the LOD of $0.68 \mathrm{~ms} / \mathrm{cy}$. With account of the various recent estimates of the geophysical effects (cf. Table 1), we find a resulting change of the LOD of $0.72 \mathrm{~ms} / \mathrm{cy}$. This value is close to that given by the observations of lunar occultations $(1.09 \mathrm{~ms} / \mathrm{cy})$ from 1680 to 2020 (black line in Fig. 5).

In the above considerations, we have used the Hubble constants at the local and cosmic scales to be the same. However, in principle, it could be conceived that the local Hubble constant might be somewhat different from the one at the global scale (e.g., by $10-20 \%$ ), for more details, the reader is referred to the works by Dumin (2008), Krizek et al. (2015), Dumin (2018), Krizek et al. (2021). Allowing such freedom in the value of the local Hubble expansion could be utilized to fit the data better.

On the whole, it appears that the LOD and LLR data may admit the occurrence of an additional contribution to the lunar recession, of the order the cosmological expansion, if we consider the results from the lunar occultations over the last 340 years, alternatively there may be an unknown source of acceleration of the Earth's rotation. Also, this is consistent with the fact that the physical action of the tidal forces on the Earth's mantle, hydrosphere, and atmosphere can only account for about $55 \%$ of the observed lunar recession (Krizek et al. 2015).

\section{Conclusions}

The observations of lunar occultations completed by the IERS data show for the period from 1680 to 2020 AD a variation rate of the LOD equal to $1.09 \mathrm{~ms} / \mathrm{cy}$. This rate is in agreement with the results of McCarthy and Babcock (1986) and Sidorenkov (2005). The above rate is lower than the mean of $1.78 \mathrm{~ms} / \mathrm{cy}$ derived on the basis of the data for eclipses from the Antiquity to $1600 \mathrm{AD}$ by Stephenson et al. (2016). We also noticed that the difference in the two rates appear at the epoch of a major change in the data accuracy with telescopic observations.

The comparison made in Fig. 5 shows first that the hypothesis of a lunar recession of $3.83 \mathrm{~cm} / \mathrm{yr}$ entirely due to tidal effects meets some difficulty with the LOD observations, whether from eclipses in the Antiquity (as also mentioned by Stephenson et al. (2020)) or from lunar occultations over the last 340 years. Secondly, when due account is also given to the recent detailed studies on the atmospheric effects, the melting from icefields, the changes of the sea level, the glacial isostatic adjustment and the core-mantle coupling, the agreement between the LOD predictions and observations is equal or better for the case of lunar occultations and the occurrence of a cosmological expansion, than for the case of antique eclipses and no cosmological effect (cf. Table 1). This is in agreement with some results by $\mathrm{Du}-$ $\min (2007,2008,2016)$.

The present study clearly shows how rich and convoluted is the subject of the LOD variations and that there are still uncertainties in geophysical processes, in particular concerning the core-mantle coupling. Thus, we do not claim this work gives a proof of the cosmological expansion at small scales, but we may say that this old question is still open.

Acknowledgements Thanks are expressed to Dr. Wolfgang Dick for providing information on the LOD from the IERS. A. M. expresses his deep gratitude to his wife and to D. Gachet for their continuous support. V. G. is extremely grateful to his wife and daughters for their understanding and family support. We are particularly grateful and highly appreciate the in-depth reading and careful reviewing of our paper by the referee, who has made many remarks that have improved the quality of the paper.

Funding Note This research received no external funding. Open access funding provided by University of Geneva.

Data availability The datasets underlaying this article were derived from sources in the public domain:

https://www.iers.org/IERS/EN/DataProducts/EarthOrientationData/ eop.html.

https://www.iers.org/IERS/EN/Science/EarthRotation.

https://astro.ukho.gov.uk/nao/lvm.

Conflict of interest The authors declare no conflict of interest.

Publisher's Note Springer Nature remains neutral with regard to jurisdictional claims in published maps and institutional affiliations.

Open Access This article is licensed under a Creative Commons Attribution 4.0 International License, which permits use, sharing, adaptation, distribution and reproduction in any medium or format, as long as 
you give appropriate credit to the original author(s) and the source, provide a link to the Creative Commons licence, and indicate if changes were made. The images or other third party material in this article are included in the article's Creative Commons licence, unless indicated otherwise in a credit line to the material. If material is not included in the article's Creative Commons licence and your intended use is not permitted by statutory regulation or exceeds the permitted use, you will need to obtain permission directly from the copyright holder. To view a copy of this licence, visit http://creativecommons.org/licenses/by/4.0/.

\section{References}

Anderson, J.L.: Phys. Rev. Lett. 75, 3602 (1995)

Balbinot, R., Bergamini, R., Comastri, A.: Phys. Rev. D 38, 2415 (1988)

Bonnor, W.B.: Gen. Relativ. Gravit. 32, 1005 (2000)

Bouvier, P., Maeder, A.: Astrophys. Space Sci. 54, 497 (1978)

Buffett, B.A.: Core-mantle interactions. In: Schubert, G. (ed.) Treatise on Geophysics, vol. 8, p. 213. Elsevier, Amsterdam (2015)

Canuto, V., Adams, P.J., Hsieh, S.-H., Tsiang, E.: Phys. Rev. D 16, 1643 (1977)

Capozziello, S., de Laurentis, M.: Phys. Rep. 509, 167 (2011)

Capozziello, S., Stornaiolo, C.: Int. J. Geom. Methods Mod. Phys. 5, 185 (2008)

Capozziello, S., Cardone, V.F., Troisi, A.: J. Cosmol. Astropart. Phys. 1706, 044 (2006)

Carrera, M., Giulini, D.: Rev. Mod. Phys. 82, 169 (2010)

Cheng, M.K., Shum, C.K., Tapley, B.D.: J. Geophys. Res. 102, 22377 (1997)

Cheng, M.K., Tapley, B.D., Ries, J.C.: J. Geophys. Res., Solid Earth 118, 740 (2013)

Christodoulidis, D.C., Smith, D.E., Williamson, R.G., et al.: J. Geophys. Res. 93(B6), 6216 (1988)

Cooperstock, F.I., Faraoni, V., Vollick, D.N.: Astrophys. J. 503, 61 (1998)

Creager, K.S.: Science 278, 1284 (1997)

Dalmau, W.: Surv. Geophys. 18, 213 (1997)

Defraigne, P., Smits, I.: Geophys. J. Int. 139, 563 (1999)

Dehant, V., Mathews, M.P.: In: Herring, T., Schubert, J. (eds.) Geodesy. Treatise of Geophysics, vol. 3, p. 295. Elsevier, Oxford (2009)

Deines, S.D., Williams, C.A.: Astron. J. 151, 103 (2016)

Deuss, A.: Annu. Rev. Earth Planet. Sci. 42, 103 (2014)

Dickey, J.O., Bender, P.L., Faller, J.E., et al.: Science 265, 482 (1994)

Dirac, P.A.M.: Proc. R. Soc. Lond. Ser. A 333, 403 (1973)

Dumberry, M., Bloxham, J.: Geophys. J. 165, 32 (2006)

Dumin, Yu.V.: Using the lunar laser ranging technique to measure the local value of hubble constant. Geophys. Res. Abstr. v, 3, p.1965 (2001)

Dumin, Yu.V.: Adv. Space Res. 31, 2461 (2003)

Dumin, Yu.V.: Testing the "Dark-Energy"-Dominated Cosmology via the Solar System Experiment (2005). arXiv:astro-ph/0507381

Dumin, Yu.V.: Phys. Rev. Lett. 98, 059001 (2007)

Dumin, Yu.V.: In: Kleinert, H., Jantzen, R., Ruffini, R. (eds.) Proc. 11th Marcel Grossmann Meeting on General Relativity, p. 1752. World Sci, ??? (2008)

Dumin, Yu.V.: In: Krizek, M., Dumin, Yu. (eds.) Cosmology on Small Scales, p. 23 (2016). Inst. of Math. Prague

Dumin, Yu.V.: Is the Hubble Constant scale-dependent? Gravit. Cosmol. 24, 171 (2018)

Dumin, Yu.V.: Lambda perturbations of Keplerian orbits in the expanding universe. Gravit. Cosmol. 26, 307 (2020)

Einstein, A., Straus, E.G.: Rev. Mod. Phys. 17, 120 (1945)

Eubanks, T.M., Steppe, J.A., Dickey, J.O., et al.: J. Geophys. Res. 90, 5385 (1985)
Faraoni, V., Jacques, A.: Phys. Rev. D 76, 3510 (2007)

Glane, S., Buffett, B.: Front. Earth Sci. (2018). https://doi.org/10.3389/ feart.2018.00171

Goldstein, S.J.: Astrophys. J. 90, 1900 (1985)

Gonzales, G.: Mon. Not. R. Astron. Soc. 470, 1436 (2018)

Gross, R.: Phys. Earth Planet. Inter. 127, 65 (2002)

Gross, R.: Earth rotation variations-long period. In: Schubert, G. (ed.) Geodesy. Treatise of Geophysics, vol. 3, p. 239. Elsevier, Amsterdam (2009)

Gross, R.: Laser ranging to Earth rotation studies. In: 20th Intnl. Worksop on Laser Ranging (2016). See https://cddis.nasa.gov/lw20/ docs/2016/presentations/05-Gross_presentation.pdf

Gueorguiev, V.G., Maeder, A.: Geometric justification of the fundamental interaction fields for the classical long-range forces. Symmetry 2021(13), 379 (2021). https://doi.org/10.3390/ sym 13030379

Hay, C.C., Morrow, E., Kopp, R.E., et al.: Nature 517, 481 (2015)

Hay, C., Mitrovica, J.X., Morrow, E., et al.: Earth Planet. Sci. Lett. 448, 115 (2016)

Herald, D., Gault, D.: Centre de Données Astronomique de Strasbourg (2012). See VI/132B at http://cdsarc.u-strasbg.fr/viz-bin/Cat

Herring, T.A.: In: International VLBI Service for Geodesy and Astronomy, 2000, General Meeting Proc., NASA/CP-2000-209893, p. $62(2000)$

Herring, T.: In: Schubert, G. (ed.) Geodesy. Treatise on Geophysics vol. 3. Elsevier, Amsterdam (2007). 445 p.

Hide, R.: Philos. Trans. R. Astron. Soc. Lond. 328, 351 (1989)

Holme, R.: Geophys. J. Int. 132, 167 (1998)

Jackson, A., Bloxham, J., Gubbins, D.: Dynamics of Earth's deep interior and Earth rotation. In: Le Mouel, J.-L., et al. (eds.) American Geophysical Union Geophysical Monograph Series, vol. 72, p. 97 (1993). Washington

Jault, D., Gire, C., Le Mouel, J.-L.: Nature 333, 353 (1988)

Jault, D.: In: Jones, C.A., et al. (eds.) Earth's Core and Lower Mantle, p. 56. Taylor and Francis, London (2003)

Johnston, P., Lambeck, K.: Geophys. J. Int. 136, 537 (1999)

Kennett, B.L.N., Engdahl, E.R., Buland, R.: Geophys. J. Int. 122, 108 (1995)

Klioner, S.A., Soffel, M.H.: In: Proc. of Symp.: The ThreeDimensional Universe with Gaia, Paris, ESA SP-576, p. 305 (2005)

Korte, M., Constable, C.G.: Continuous geomagnetic field models for the past 7 millennia: 2. CALS7K. Geochem. Geophys. Geosyst. 6, Q02H16 (2005). https://doi.org/10.1029/2004GC000801

Kottler, F.: Ann. Phys. 56, 401 (1918)

Krizek, M., Gueorguiev, V.G., Maeder, A.: An Alternative Explanation of the Rapid Orbital Expansion of Titan (2021). Preprints.org, arXiv:202109.0200

Krizek, M., Krizek, F., Somer, L.: Antigravity-Its Origin and Manifestations, Lambert Academic Publishing, xiv+348 pp., ISBN 978-3-659-79834-4

Kuang, W., Chao, B.F., Chen, J.: Geodes. Geodyn. 10, 356 (2018)

Lainey, V., Casajus, L.G., Fuller, J., et al.: Resonance locking in giant planets indicated by the rapid orbital expansion of Titan. Nat. Astron. 4, 1053-1058 (2020)

Maeder, A.: Astrophys. J. 834, 194 (2017)

Maeder, A., Gueorguiev, V.G.: Scale-invariant dynamics of galaxies, MOND, dark matter, and the dwarf spheroidals. Mon. Not. R. Astron. Soc. 492, 2698 (2020a). 2001.04978

Maeder, A., Gueorguiev, V.G.: Universe 6, 46 (2020b)

McCarthy, D.D., Babcock, A.: Phys. Earth Planet. Inter. 44, 281 (1986)

McVittie, G.C.: Condensations in an expanding universe. Mon. Not. R. Astron. Soc. 92, 500 (1932)

McVittie, C.G.: Mon. Not. R. Astron. Soc. 93, 325 (1933)

Meierovich, B.E.: Phys. Rev. D 85, 123544 (2012) 
Meierovich, B.E.: Phys. Rev. D 87, 103510 (2013)

Milgrom, M.: Astrophys. J. 270, 365 (1983)

Milgrom, M.: Phys. Rev. D 99, 04404 (2019)

Misner, C.W., Thorne, K.S., Wheeler, J.A.: Gravitation. Freeman and Co., San Francisco (1973). 1279 p.

Mitrovica, J.X., Forte, A.M.: J. Geophys. Res. 102(B2), 2751 (1997)

Mitrovica, J.X., Forte, A.M.: Earth Planet. Sci. Lett. 225, 177 (2004)

Mitrovica, J.X., Wahr, J.: Annu. Rev. Earth Planet. Sci. 39, 577 (2011)

Mitrovica, J.X., Milne, G.A., Davis, J.L.: Geophys. J. Int. 147, 562 (2001)

Mitrovica, J.X., Hay, C.C., Morrow, E., et al.: Sci. Adv. 1, 1500679 (2015)

Munk, W.H.: Proc. Natl. Acad. Sci. USA 99, 6550 (2002)

Munk, W.H., MacDonald, G.J.F.: The Rotation of the Earth: A Geophysical Discussion. Cambridge University Press, Cambridge (1960). 323 p.

Murphy, T.W., Adelberger, E.G., Battat, J.B.R., et al.: Icarus 211, 1103 (2011). https://doi.org/10.1016/j.icarus.2010.11.010

Nakada, M., Okuno, J.: Geophys. J. Int. 152, 124 (2003)

Nerem, R.S.B.F., Chao, A.Y., Au, J.C., et al.: Geophys. Res. Lett. 20, 595 (1993)

Novotny, O.: Motions, Gravity Field and Figure of the Earth. Lecture Notes. Univ. Fedda, Bahia, Brazil (1998)

Olson, P.: In: Schubert, G. (ed.) Core Dynamics. Treatise on Geophysics, vol. 8. Elsevier, Amsterdam (2007). 357 p.

Peltier, W.R.: Rev. Geophys. 36, 603 (1998)

Peltier, W.R.: Glob. Planet. Change 20, 93 (1999)

Peltier, W.R.: Annu. Rev. Earth Planet. Sci. 32, 111 (2004)

Peltier, W.R.: History of the Earth rotation. In: Schubert, G. (ed.) Evolution of the Earth. Treatise on Geophysics, vol. 9. Elsevier, Amsterdam (2007)

Ponsar, S., Dehant, V., Van Hoolst, T.: Proc. IERS Workshop on Combination Research and Global Geophysical Fluids, Bavarian Acad.of Sci. Munich, IERS TechNote No. 30 (2003)

Price, R.H., Romano, J.D.: (2005). arXiv:gr-qc/0508052

Radok, U., Jenssen, D., McInnes, B.: Publ. DOE/ER/60197-HI, National Techn. Info. Service, US Dept. of Commerce, Springfield, VA, USA (1987)

Ray, R.D., Egbert, G.D.: Geophys. J. Int. 189, 400 (2012)

Roberts, P.H., Aurnou, J.M.: Geophys. Astrophys. Fluid Dyn. 106, 2 (2012)

Rubincam, D.P.: Gravitational core-mantle coupling and the acceleration of the Earth. J. Geophys. Res. 108(B7), 2338 (2003). https:// doi.org/10.1029/2002JB002132

Schindelegger, M., Saltein, D., Einspiegel, D., et al.: Geophys. Res. Lett. 44, 2755 (2017)

Sereno, M., Jetzer, P.: Phys. Rev. D 75, 064031 (2007)

Shiga, D.: Space News \& Technology (April 27, 2010): Long-lost lunar rover successfully zapped with laser, New Scientist, issue 2758 (1 May 2010). https://www.newscientist.com/article/dn18826-longlost-lunar-rover-successfully-zapped-with-laser/

Shuanggen, J.: Planetary Geodesy and Remote Sensing. CRC Press, Boca Raton (2014). 396 p.
Sidorenkov, N.S.: Astron. Rep. 41, 705 (1997)

Sidorenkov, N.S.: Physics of the Earth's rotation instabilities. Astron. Astrophys. Trans. 24, 425 (2005)

Sidorenkov, N.S.: Odessa Astron. Publ. 29, 196 (2016)

Simon, B., Lemaitre, A., Souchay, J.: In: Souchay, J., Mathis, S., Tokieda, T. (eds.) Tides in Astronomy and Astrophysics. Lecture Notes in Physics, vol. 861, p. 83. Springer, Heidelberg (2013)

Sola, J., Gomez-Valent, A., de Cruz Perez, J.: Astrophys. J. 811, L14 (2015)

Sola, J., Gomez-Valent, A., de Cruz Perez, J.: Mod. Phys. Lett. 32, 1750054 (2017)

Soma, M., Tanikawa, K.: Publ. Astron. Soc. Jpn. 68, 29 (2016)

Sosnik, K., Jaggi, A., Thaller, D., et al.: Earth rotation and gravity field parameters from satellite laser ranging (2016). See http://cddis. nasa.gov/lw19/docs/2014/Papers/3072_Sosnica_paper.pdf

Souriau, A., Calvet, M.: In: Schubert, G. (ed.) Treatise of Geophysics, p. 725 (2015)

Stephenson, F.R.: Historical Eclipses and Earth's Rotation. Cambridge University Press, Cambridge (1997). 554 p.

Stephenson, F.R., Morrison, L.V.: Philos. Trans. R. Soc. Lond. A 313, 207 (1984)

Stephenson, F.R., Morrison, L.V.: Philos. Trans. Phys. Sci. Eng. 351, 165 (1995)

Stephenson, F.R., Morrison, L.V., Hohenkerk, C.Y.: Proc. R. Soc. A 472, 20160404 (2016)

Stephenson, F.R., Morrison, L.V., Hohenkerk, C.Y.: IAU XXX General Assembly. In: Proceedings of the IAU, p. 160 (2020)

Sung-Ho, N., Jungho, C., Tu-Hwan, K., et al.: J. Astron. Space Sci. 33, 295 (2016)

Thompson, L.G., Davis, M.E., Mosley-Thompson, E., Porter, S.E., Corrales, G.V., Shuman, C.A., Tucker, C.J.: The impacts of warming on rapidly retreating high-altitude, low-latitude glaciers and ice core-derived climate records. Glob. Planet. Change 203, 103538 (2021)

Tokieda, T.: In: Souchay, J., Mathis, S., Tokieda, T. (eds.) Tides in Astronomy and Astrophysics. Lecture Notes in Physics, vol. 861, p. 1. Springer, Heidelberg (2013)

Trupin, A.S.: Geophys. J. Int. 113, 273 (1993)

Tsuboi, S., Butler, R.: Phys. Earth Planet. Inter. 301, 106451 (2020)

van Flandern, T.C.: Mon. Not. R. Astron. Soc. 170, 333 (1975)

Vidale, J.E.: Geophys. Res. Lett. 46, 9483 (2019)

Williams, J.G.: Astron. J. 108, 711 (1994)

Williams, J.G., Boggs, D.H.: Celest. Mech. Dyn. Astron. 126, 89 (2016)

Williams, J.G., Boggs, D.H., Yoder, C.F., Ratcliff, J.T., Dickey, J.O.: Lunar rotational dissipation in solid body and molten core. J. Geophys. Res. 106(E11), 27933-27968 (2001). https://doi.org/10. 1029/2000JE001396

Williams, J.G., Turyshev, S.G., Boggs, D.H.: Phys. Rev. Lett. 93, 261101 (2004)

Williams, J.G., Boggs, D.H., Ratcliff, J.T.: 47th Lunar and Planetary Science Conference p. 1096 (2016)

Yoder, C.F., Williams, J.G., Dickey, J.O., et al.: Nature 303, 757 (1983) 